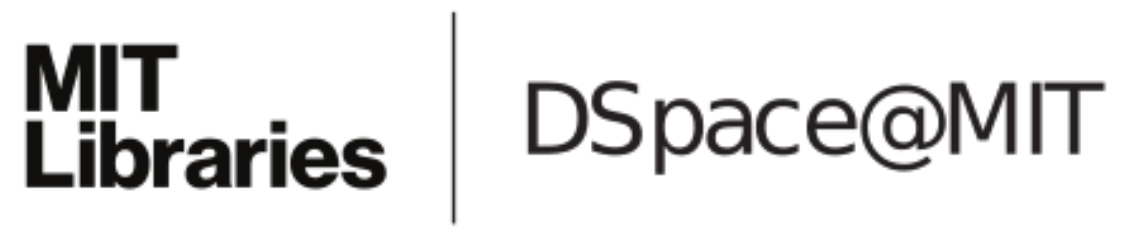

\author{
MIT Open Access Articles
}

Analysis and Control of a Variable-Pitch Quadrotor for Agile Flight

The MIT Faculty has made this article openly available. Please share how this access benefits you. Your story matters.

Citation: Cutler, Mark and How, Jonathan P. "Analysis and Control of a Variable-Pitch Quadrotor for Agile Flight." Journal of Dynamic Systems, Measurement, and Control 137, no. 10 (July 2015): 101002. () 2015 American Society of Mechanical Engineers

As Published: http://dx.doi.org/10.1115/1.4030676

Publisher: American Society of Mechanical Engineers

Persistent URL: http://hdl.handle.net/1721.1/109136

Version: Final published version: final published article, as it appeared in a journal, conference proceedings, or other formally published context

Terms of Use: Article is made available in accordance with the publisher's policy and may be subject to US copyright law. Please refer to the publisher's site for terms of use. 


\author{
Mark Cutler \\ Aerospace Controls Laboratory, \\ Department of Aeronautics and Astronautics, \\ Massachusetts Institute of Technology, \\ Cambridge, MA 02139 \\ e-mail: cutlerm@mit.edu \\ Jonathan P. How \\ Richard Maclaurin Professor \\ of Aeronautics and Astronautics \\ Aerospace Controls Laboratory, \\ Department of Aeronautics and Astronautics, \\ Massachusetts Institute of Technology, \\ Cambridge, MA 02139 \\ e-mail: jhow@mit.edu
}

\section{Analysis and Control of a Variable-Pitch Quadrotor for Agile Flight}

Fixed-pitch quadrotors are popular research and hobby platforms largely due to their mechanical simplicity relative to other hovering aircraft. This simplicity, however, places fundamental limits on the achievable actuator bandwidth and the possible flight maneuvers. This paper shows that many of these limitations can be overcome by utilizing variable-pitch propellers on a quadrotor. A detailed analysis of the potential benefits of variable-pitch propellers over fixed-pitch propellers for a quadrotor is presented. This analysis is supported with experimental testing to show that variable-pitch propellers, in addition to allowing for generation of reverse thrust, substantially increase the maximum rate of thrust change. A nonlinear, quaternion-based control algorithm for controlling the quadrotor is also presented with an accompanying trajectory generation method that finds polynomial minimum-time paths based on actuator saturation levels. The control law and trajectory generation algorithms are implemented on a custom variable-pitch quadrotor. Several flight tests are shown, which highlight the benefits of a variable-pitch quadrotor over a standard fixed-pitch quadrotor for performing aggressive and aerobatic maneuvers. [DOI: 10.1115/1.4030676]

\section{Introduction}

Small, multirotor helicopters equipped with electric motors and fixed-pitch propellers have gained popularity as experimental and hobby platforms over the past 10-12 years. Fixed-pitch, multirotor designs are mechanically simple as they lack the complexity of the control linkages and swashplate that are required by traditional pod-and-boom style helicopters.

The mechanical simplicity and robustness of fixed-pitch, multirotor vehicle designs, however, places fundamental limits on the achievable flight performance of the vehicle. With fixed-pitch propellers and commonly utilized brushless motors and electronic speed controllers (ESCs), thrust can only be generated in one direction, thus preventing the multirotor vehicle from generating upward thrust (with respect to the vehicle body). Also, the attainable control bandwidth with fixed-pitch propellers is limited by the inertia of the motors and propellers. These limitations could restrict the aggressive and aerobatic maneuvers a multirotor helicopter can perform.

This paper explores the benefits of adding variable-pitch propellers to an autonomous quadrotor helicopter (see Fig. 1). Variable-pitch propellers largely overcome the limitations resulting from fixed-pitch flight. Reversed thrust is achievable and control bandwidth is limited only by the speed of the variable-pitch actuation, not by the inertia of the motor-propeller combination. While variable-pitch propellers require a few additional mechanical components, the advantages of increased controller bandwidth and reverse thrust capabilities could justify the design when aggressive and agile flight is required.

\section{Related Work}

In traditional fixed-pitch quadrotors, stability and flight control are achieved by changing the voltage supplied to each of the four motors, inducing a change in the motor revolutions per minute (rpm) and, correspondingly, the thrust generated by each of the

Contributed by the Dynamic Systems Division of ASME for publication in the Journal of Dynamic Systems, Measurement, and Control. Manuscript received October 15, 2014; final manuscript received April 28, 2015; published online July 1, 2015. Assoc. Editor: Dejan Milutinovic. propellers. Several detailed descriptions of the modeling of quadrotors and their dynamics have been published recently [1-4]. Considerable work also exists on various control schemes for controlling quadrotors [5-8]. Furthermore, trajectory generation for quadrotors has been considered, with several results showing time-optimal trajectory generation methods and corresponding tracking algorithms [9-12].

Controller bandwidth can be a significant problem for quadrotors, becoming an issue for quadrotor stability as the size of the quadrotor increases [13]. Larger quadrotors require larger motors which, in turn, have larger inertias and cannot be controlled as quickly as smaller motors. Eventually, as the rotor size increases enough (rotor inertia scales with rotor radius to the fifth power $[14,15])$, the quadrotor can no longer be stabilized through rpm control alone. The torque required to change the rotational velocity of the motor exceeds the capacity of the motor and the power supply. Thus, variable-pitch blades may become necessary for larger quadrotors merely for stabilization purposes.

A few variable-pitch quadrotors have previously been developed. The HoverBot utilized variable-pitch propellers and electric

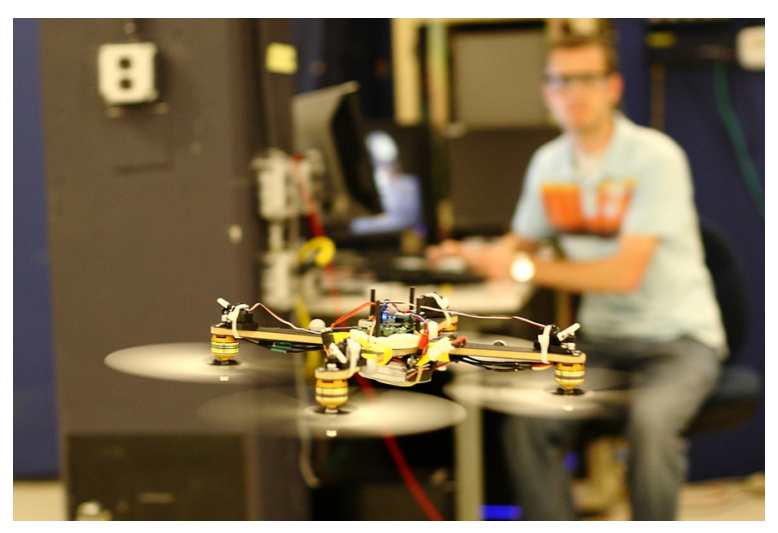

Fig. 1 The variable-pitch quadrotor during inverted flight. The symmetry introduced by the variable-pitch propellers allows the quadrotor to fly equally well upright or inverted. 
motors. The vehicle achieved promising results but was never able to fly autonomously [16]. Several hobbyists have developed, and are currently developing, remote-controlled variable-pitch quadrotors, and are posting their results on online forums and discussion groups (see, e.g., Ref. [17]). Finally, concurrent with the initial design work for this project [18], the Vehicle Control Systems Laboratory at the National Cheng Kung University developed a variable-pitch quadrotor that demonstrated autonomous upright and inverted flight and flips [19], but no further work appears to have been published on the project. This paper also verifies the feasibility of an autonomous variable-pitch quadrotor, but focuses mainly on the analysis of the potential benefits of variable-pitch propellers for quadrotors, and on the implemented control and trajectory generation algorithms.

Several research groups have successfully demonstrated autonomous indoor aerobatics with fixed-pitch quadrotors, including triple flips with rotational rates exceeding $1600 \mathrm{deg} / \mathrm{s}$ [20]. Also, fixed-pitch quadrotors have been shown to fly through windows, perch on inverted surfaces [21], fly through moving hoops [11], juggle and throw balls [22,23], hold inverted pendulums [24], and perform other aggressive, agile, and aerobatic tasks. These aerobatic and aggressive maneuvers, however, lag significantly behind the abilities of single-rotor helicopters [25], primarily because fixed-pitch multirotor helicopters lack the ability to generate negative thrust. This substantially hinders the flight envelope of the vehicle, making maneuvers such as $180 \mathrm{deg}$ flips, tic-toc's, and split-s' impossible. Also, the fixed-pitch propellers make decelerating faster than gravity difficult. While fast decelerations have been demonstrated with fixed-pitch quadrotors, they require flipping the quadrotor upside down to generate the required force. The flipping behavior is only beneficial if both the required decelerations and vertical displacements are large [10].

2.1 Overview. The purpose of this paper is to demonstrate how adding variable-pitch propellers to a quadrotor overcomes some of the flight limitations of fixed-pitch quadrotors, specifically in the area of autonomous aggressive and aerobatic flight. The main contributions of this work are threefold:

(1) A theoretical and empirical comparison of variable-pitch versus fixed-pitch propellers for quadrotors.

(2) Development of control and trajectory generation algorithms for a variable-pitch quadrotor, with practical robustness demonstrated through real-world testing.

(3) Flight experiments conducted on a custom variable-pitch quadrotor, which verify the analytical results.

Much of the work presented in this paper has been introduced earlier [18,26,27], with several new experimental results presented in this paper.

The remainder of the paper is organized as follows: Sec. 3 analyzes the dynamic differences in thrust output between a fixedpitch and variable-pitch propeller. Control and trajectory generation algorithms for a quadrotor helicopter are presented in Sec. 4. Section 5 briefly discusses the design of a custom variable-pitch quadrotor built at the Aerospace Controls Laboratory (ACL). Experimental results on the variable-pitch quadrotor demonstrating the control design and trajectory generation method are given in Sec. 6. These results validate the predicted performance increase of utilizing variable-pitch propellers over fixed-pitch propellers.

\section{Actuator Comparison}

With fixed-pitch propellers, given a constant motor rotational rate, the thrust produced by the propeller is constant (assuming that the quadrotor is near hover). The only way to change the thrust produced by the propeller is by changing the voltage to the motor, thereby inducing a change in the rotational rate of the propeller.

Adding variable-pitch propellers to the quadrotor platform results in an additional degree-of-freedom for varying the thrust produced by each motor-propeller combination. With variable-pitch propellers, thrust can be changed by either changing the blade pitch or by changing the rotational rate of the motors. These two actuators, to a large extent, overlap. For instance, with variable-pitch propellers, a quadrotor can hover by spinning the propeller quickly and with a low blade pitch, or by slowing the rotational rate of the motor and increasing the blade pitch. There are many combinations of motor speed and propeller pitch that yield identical thrust values. The number of possible combinations is only limited by the maximum propeller pitch (physical and aerodynamic limitations), the maximum available motor power, and the available discretization in the hardware motor and pitch commands.

For the purposes of this analysis, the output of the quadrotor control algorithm is assumed to be a desired thrust for each of the four motor/propeller combinations (the details of the control algorithms will be presented in Sec. 4). This section addresses the benefits of adding variable-pitch control to a quadrotor while considering the allocation problem of which actuator to use, pitch, or motor speed, when a given thrust value is desired.

3.1 Motor-Propeller Model. Using a simple first-order direct current (DC) motor model [28], the propeller speed, $\omega$, is related to the voltage applied to the motor, $v$, the motor voltage and torque constants, $K_{\mathrm{V}}$ and $K_{\mathrm{Q}}$, the motor internal resistance, $R$, the no-load current, $i_{0}$, the inertia of the motor and propeller, $I$, and the drag, $D$, by

$$
I \dot{\omega}=\left[\left(v-\frac{\omega}{K_{\mathrm{V}}}\right) \frac{1}{R}-i_{0}\right] \frac{1}{K_{\mathrm{Q}}}-D
$$

This simple motor model assumes a brushed DC motor and therefore is only an approximation for the brushless motors used on the variable-pitch quadrotor. Substantially, more detailed analyses and full derivations of the equations for each of the phases of a brushless motor have been shown in Refs. [29,30], where the developed equations show that each phase of a brushless motor can be approximately modeled as a brushed DC motor and thus, when averaged, the three phases approximately follow Eq. (1). Table 2 shows the motor coefficients for the motors used on the variable-pitch quadrotor in this paper.

Assuming the quadrotor is near hover, the lift, L, and drag, D, generated by the propeller are modeled by [31]

$$
\begin{gathered}
L=\rho c R_{\mathrm{p}}^{3} \omega^{2} C_{\mathrm{L} \alpha} \frac{\alpha}{3} \\
D=\rho c R_{\mathrm{p}}^{4} \omega^{2}\left(\frac{C_{\mathrm{D} 0}+C_{\mathrm{D} i} \alpha^{2}}{4}-\frac{C_{\mathrm{L} \alpha} \alpha}{3 R_{\mathrm{p}} \omega}\right)
\end{gathered}
$$

where $\alpha$ is the propeller pitch angle, and $\rho, c, R_{\mathrm{p}}, C_{\mathrm{D} 0}, C_{\mathrm{D} i}$, and $C_{\mathrm{L} \alpha}$ are constants relating to the physical and aerodynamic properties of the propellers and surrounding air. Combining the constants and calling them $b_{\mathrm{L}}, b_{\mathrm{D}_{1}}, b_{\mathrm{D}_{2}}$, and $b_{D_{3}}$, Eqs. (2) and (3) are rewritten as

$$
\begin{gathered}
L=b_{\mathrm{L}} \omega^{2} \alpha \\
D=b_{\mathrm{D}_{1}} \omega^{2}+b_{\mathrm{D}_{2}} \omega^{2} \alpha^{2}+b_{\mathrm{D}_{3}} \omega \alpha
\end{gathered}
$$

Substituting Eq. (5) into Eq. (1) results in the following nonlinear differential equation for $\dot{\omega}$ :

$$
\begin{aligned}
I \dot{\omega}= & {\left[\left(v-\frac{\omega}{K_{\mathrm{V}}}\right) \frac{1}{R}-i_{0}\right] \frac{1}{K_{\mathrm{Q}}} } \\
& -b_{\mathrm{D}_{1}} \omega^{2}-b_{\mathrm{D}_{2}} \omega^{2} \alpha^{2}-b_{\mathrm{D}_{3}} \omega \alpha
\end{aligned}
$$

Linearizing these equations about the hover conditions $\omega_{0}$ and $\alpha_{0}$ results in the state-space system 


$$
\begin{aligned}
& \Delta \dot{\omega}=-\frac{1}{I}\left[\frac{1}{R K_{\mathrm{V}} K_{\mathrm{Q}}}+2 b_{\mathrm{D}_{1}} \omega_{0}+2 b_{\mathrm{D}_{2}} \omega_{0} \alpha_{0}^{2}+b_{\mathrm{D}_{3}} \alpha_{0}\right] \Delta \omega \\
& +\frac{1}{I}\left[\frac{1}{R K_{\mathrm{Q}}} \quad-2 b_{\mathrm{D}_{2}} \omega_{0}^{2} \alpha_{0}-b_{\mathrm{D}_{3}} \omega_{0}\right]\left[\begin{array}{c}
\Delta v \\
\Delta \alpha
\end{array}\right] \\
& \Delta L=\left[2 b_{\mathrm{L}} \omega_{0} \alpha_{0}\right] \Delta \omega+\left[\begin{array}{ll}
0 & b_{\mathrm{L}} \omega_{0}^{2}
\end{array}\right]\left[\begin{array}{c}
\Delta v \\
\Delta \alpha
\end{array}\right]
\end{aligned}
$$

These linearized equations encapsulate one of the fundamental differences between variable-pitch and fixed-pitch actuators. With a fixed-pitch propeller, thrust is increased by increasing the voltage supply to the motor. The motor voltage is applied in Eq. (7), and first increases the motor speed which, in turn, increases the thrust output. Thus, the thrust rate of change is fundamentally limited by the dynamics of the motor. However, with a variable-pitch actuator, there is a significant nonzero direct feed-through term in the lift output shown in Eq. (8). Any change in the pitch of the blades directly affects lift, bypassing the motor dynamics. Of course, increasing the pitch of the propellers negatively impacts the speed of the motor and therefore negatively impacts the lift produced, but that decrease in lift is first filtered through the motor dynamics. Provided the mechanism for actuating pitch is fast when compared to the response of the motor, varying the pitch of the blades results in faster changes in thrust than varying the motor voltage.

3.2 Simulated Thrust Response. In this section, we provide several simulations of the motor-propeller combination used on the physical system. The simulation data are not used for control, but rather to illustrate fundamental differences between fixedpitch and variable-pitch propellers on a quadrotor helicopter.

The propellers used on the hardware are symmetric, tapered, 9 in. diameter blades. To determine the lift and drag coefficients of the propellers to use in the analysis, an airfoil, operating Reynolds number, and Mach number must be selected. The maximum thickness to chord ratio is 0.899 , and so a NACA 0009 airfoil is chosen to model the propeller airfoil. Assuming the blades are rotating at $8000 \mathrm{rpm}$ (typical for our choice of motor and propeller), the Mach number near the end of the blades is about 0.25 . This gives an operating Reynolds number of around 100,000.

Using XFOIL [32], aerodynamic coefficients for the propeller blades are determined and, for reference, are displayed in Table 1.

A propeller and motor numerical analysis program, QPROP [28], is used to determine the steady-state values of the drag and lift, generated by the propellers as functions of $\omega$ and $\alpha$. The numerical values of the drag and lift coefficients are shown in Table 3 for reference. Figures 2-4 show the output of QPROP. In each plot, lines of constant thrust are denoted in (predominantly vertical lines) and lines of constant motor speed are denoted in (predominantly horizontal lines). The shaded areas indicate operating regimes that should be avoided due to excessive motor vibrations and propeller stall. The approximate hover thrust required by each of the four motors is denoted by the green line, indicating the many combinations of pitch and voltage settings that could be used to hover. The plot also shows how thrust can be increased from hover by increasing motor voltage, increasing pitch, or by increasing both. Figure 3 shows the same information as Fig. 2, except here the horizontal axis shows both positive and negative propeller pitch. One of the key benefits of the variablepitch propeller actuator is the ability to move anywhere on this thrust plot. With fixed-pitch propellers, the vehicle is restricted to moving only vertically on this plot with a set positive propeller pitch.

Table 1 Propeller aerodynamic coefficients

\begin{tabular}{cccccccccc}
\hline \hline$C_{\mathrm{L} 0}$ & $C_{\mathrm{La}}$ & $C_{\mathrm{Lmin}}$ & $C_{\mathrm{Lmax}}$ & $C_{\mathrm{D} 0}$ & $C_{\mathrm{D} 2 \mathrm{u}}$ & $C_{\mathrm{D} 21}$ & $C_{\mathrm{L}} C_{\mathrm{D} 0}$ & $\mathrm{RE}_{\mathrm{ref}}$ & $\mathrm{RE}_{\text {exp }}$ \\
\hline 0.0 & 2.87 & 0.0 & 0.7 & 0.01 & 0.0408 & 0.0408 & 0.0 & 100,000 & -0.5 \\
\hline \hline
\end{tabular}

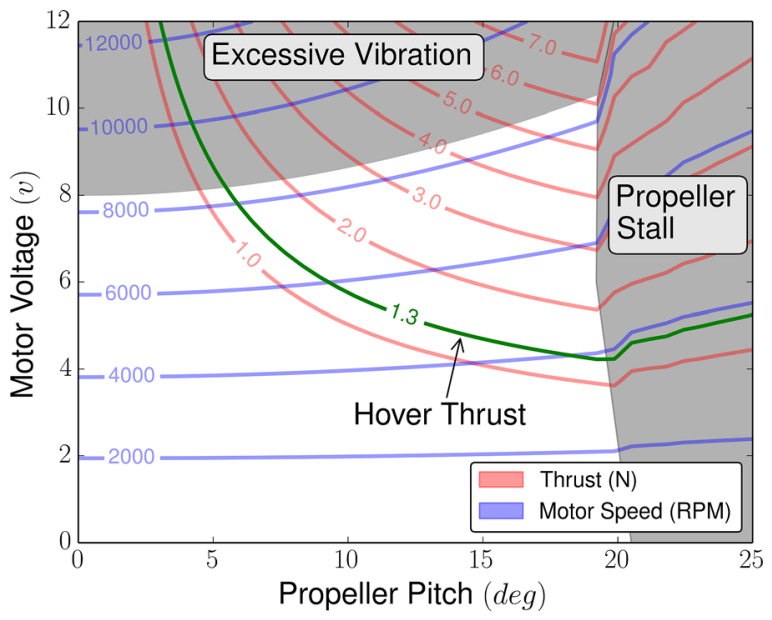

Fig. 2 Lines of constant thrust (curved lines) and constant motor speed (straight lines), as a function of motor voltage and propeller pitch. The thrust required by the vehicle to hover is indicated by the dark gray line. The addition of variable-pitch actuators has led to a continuum of possible command settings for generating specific thrust settings. Only positive propeller pitch is displayed.

In Fig. 4, the vertical axis shows power consumed by the motor as a function of propeller pitch. Another benefit of variable-pitch propellers over fixed-pitch propellers is the ability to choose more efficient operating regimes based on vehicle loading. Although not explored in this paper, Fig. 4 suggests that propeller pitch could be adjusted to more power efficient settings as the required thrust increases or decreases.

Figure 5 shows nonlinear simulation results from the data generated by QPROP for lift and motor speed responses to steps in voltage and pitch. The direct feed-through from pitch to lift is apparent as the lift quickly increases when the step in pitch is commanded. The lift then decreases as the increased torque on the motor slows the motor down to the new steady-state value.

Note that Fig. 5 also accounts for limitations in the ability to change voltage to the motor and the speed of the servo. Bench tests suggest that the dynamics of the ESC can be approximated as a rate limiter of $70 \mathrm{~V} / \mathrm{s}$. Also, on the actual quadrotor, the propeller pitch is actuated by a digital servo. The servo dynamics are approximately modeled by a rate limit and a small lag. No-load specifications on the servos indicate a rate limit of about $900 \mathrm{deg} / \mathrm{s}$. Bench

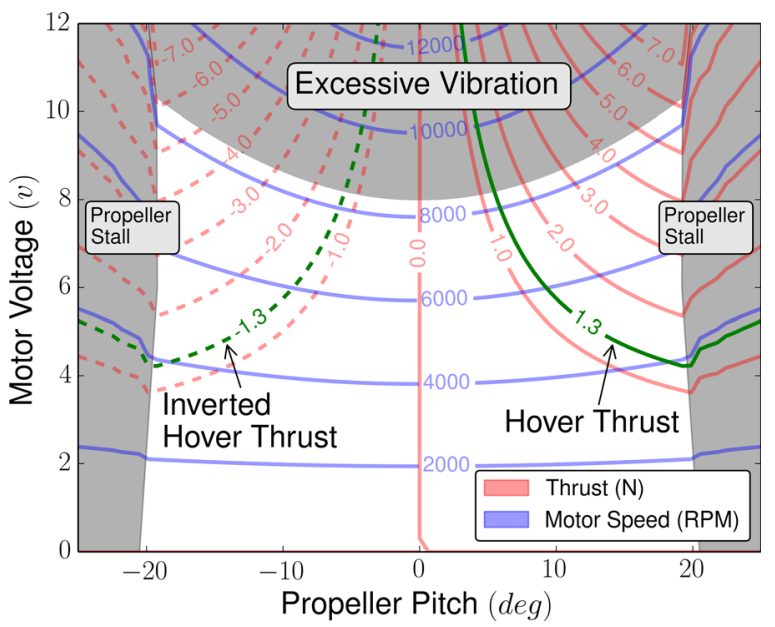

Fig. 3 Same plot as in Fig. 2 but with both positive and negative pitch shown. One of the fundamental benefits of the variable-pitch actuators for quadrotors is the addition of negative thrust to the flight regime. 


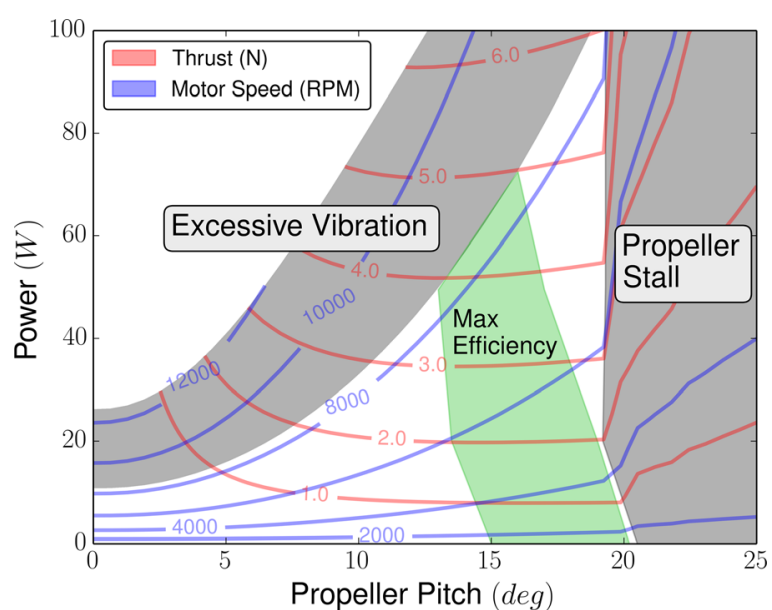

Fig. 4 Lines of constant thrust (horizontal lines) and constant motor speed (vertical lines), as a function of motor power and propeller pitch. The center shaded area roughly indicates areas of maximum efficiency, where the power is minimized for a given thrust value. Only positive propeller pitch is displayed.

test data indicate that the loaded response is roughly $70 \%$ of that value. As Fig. 5 indicates, the servo response is much faster than the dynamics of the motor. The fast actuation benefits of a variable-pitch actuator hinge on this assumption that the pitch actuator is faster than the motor dynamics.

3.3 Control Allocation. Choosing the thrust actuation mechanism (ratio of varying voltages versus varying pitches) depends on a number of factors. For instance, to minimize power consumption, Fig. 4 suggests keeping the blade pitch between 14 and 19 deg when hovering since the constant thrust lines have minimum power in this range. However, if quick thrust changes are desired, hovering with a lower pitch and higher rpm allows the propeller pitch to increase more before stalling the propellers. In other words, the motor-propeller combination contains more kinetic energy at hover that can be quickly converted into thrust by increasing the propeller pitch.

Agile and aggressive flight require quick changes in thrust [5]. It follows that for agile flight, the kinetic energy stored in the motor should be maximized. As indicated in Fig. 2, bench tests confirm that vibration from the motors becomes excessive for the frame and autopilot around $8000 \mathrm{rpm}$, placing an upper limit on the kinetic energy that can be stored in the motor. Keeping the energy high under varying pitch values implies that the power to the motor must be adjusted to compensate for the varying propeller loads (Table 2).

One strategy for agile flight is to always keep the motor speed at its upper limit. Given an initial thrust level, $L_{0}$, and maximum motor speed, $\omega_{\max }$, the required propeller pitch, $\alpha_{0}$, is found using Eq. (4). Any desired change in output thrust, $\Delta L$, is made by commanding the corresponding $\Delta \alpha$, found from Eq. (8). The motor speed is kept constant by simultaneously choosing the voltage input to compensate for subsequent changes in motor speed due to increased or decreased propeller drag.

$$
\Delta v=R K_{\mathrm{Q}}\left(2 b_{\mathrm{D}_{2}} \omega_{\max }^{2} \alpha_{0}+b_{\mathrm{D}_{3}} \omega_{\max }\right) \Delta \alpha
$$

This choice of commanded voltage effectively cancels the pole of the motor dynamics in Eq. (7) with a zero from the pitch direct feed-through term. The state-space system from Eqs. (7) and (8) becomes

$$
\Delta \dot{\omega}=-\frac{1}{I}\left[\frac{1}{R K_{\mathrm{V}} K_{\mathrm{Q}}}+2 b_{\mathrm{D}_{1}} \omega_{0}+2 b_{\mathrm{D}_{2}} \omega_{0} \alpha_{0}^{2}+b_{\mathrm{D}_{3}} \alpha_{0}\right] \Delta \omega
$$
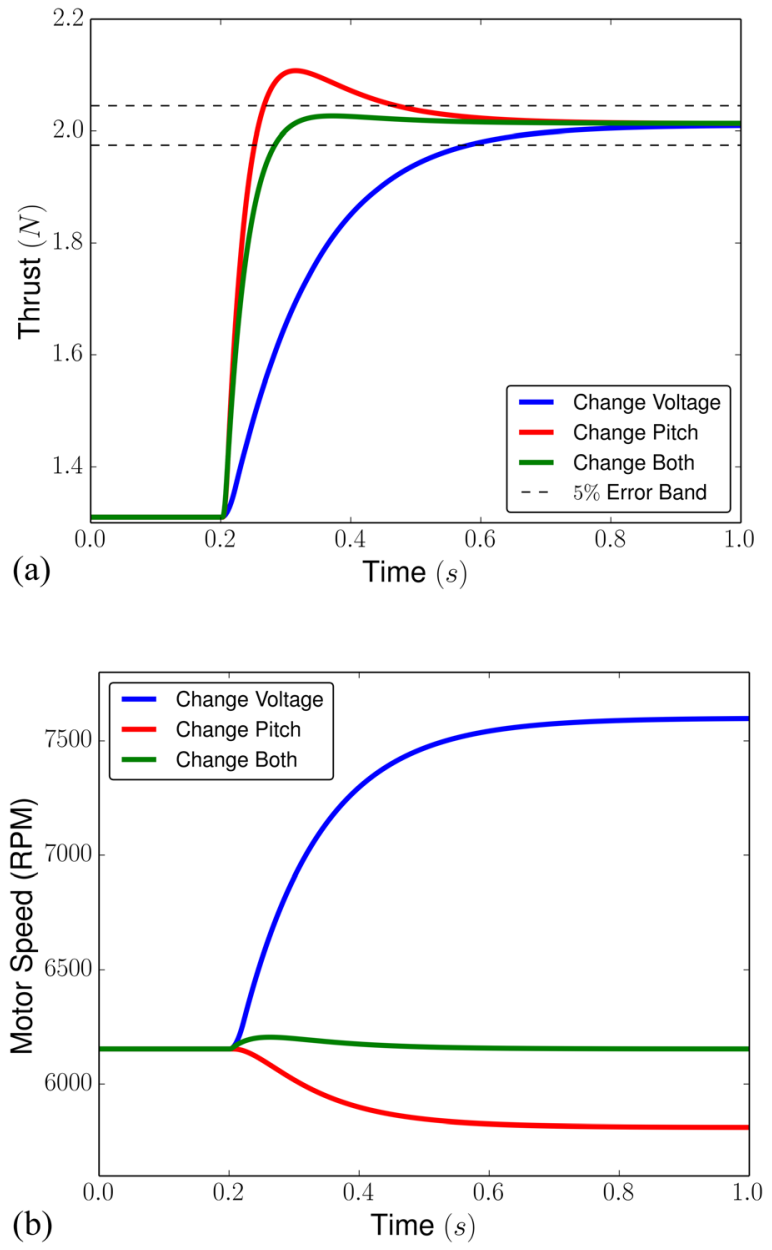

Fig. 5 Simulated thrust and motor speed responses to step increases in motor voltage and propeller pitch (the steps occur at $0.2 \mathrm{~s}$ ). Changing thrust by varying both motor speed and propeller pitch significantly reduces the effects of motor dynamics, yielding clean, fast changes in thrust. (a) Simulated thrust response to steps in voltage and pitch and $(b)$ simulated motor speed response to steps in voltage and pitch.

$$
\Delta L=\left[2 b_{\mathrm{L}} \omega_{0} \alpha_{0}\right] \Delta \omega+\left[b_{\mathrm{L}} \omega_{0}^{2}\right] \Delta \alpha
$$

showing that, using the linearized dynamics, lift and motor speed have become input decoupled. Figure 5 demonstrates that when applied to the full nonlinear motor dynamics, this strategy increases the thrust quickly while avoiding any significant rpm and thrust decrease after the increase in pitch. The mitigation of slow motor dynamics during thrust changes illustrates an advantage of using both voltage and pitch control in combination. In practice, this idea is utilized on the variable-pitch quadrotor in order to maximize the potential for agile and aggressive flights. Note that on the physical quadrotor, we are unable to implement Eq. (9) directly as we do not command the voltage sent to each motor, but rather only send nondimensional commands to the speed controllers. However, we approximate this approach by using bench data to calculate the feedforward input needed to keep the motors running at near constant rpm regardless of pitch settings.

Table 2 Motor coefficients

\begin{tabular}{lccc}
\hline \hline$K_{\mathrm{V}}(\mathrm{rad} / \mathrm{sV})$ & $K_{\mathrm{Q}}(\mathrm{A} / \mathrm{NM})$ & $R(\Omega)$ & $i_{0}(\mathrm{~A})$ \\
\hline 115.2 & 115.2 & 0.26 & 0.35 \\
\hline \hline
\end{tabular}


3.4 Bench Motor Testing. The analytical results presented in Sec. 3.3 are verified here using bench testing of one of the motors and propellers used on the variable-pitch quadrotor. The tests are performed by rigidly attaching a motor and propeller to a load cell. Static tests show a maximum and minimum safe thrust value of about $3 \mathrm{~N}$ and $-3 \mathrm{~N}$ per motor, respectively. When the pitch is locked to a positive value (simulating a fixed-pitch propeller), the minimum thrust value increases to about $0.15 \mathrm{~N}$.

Figure 6 shows the results of dynamic tests where various step inputs in commanded motor thrust and propeller pitch are given. While the decreases in thrust and motor speed after the initial command given are greater than predicted by the simulations in Fig. 5, the overall behavior of the system agrees with the simulated data. When increasing the motor command and keeping the pitch constant, the time constant of the thrust response is governed by the inertia of the motor and propeller. On the other hand, when pitch is increased with a constant motor command, the thrust changes nearly instantaneously, followed by a decrease in thrust as the increased propeller drag slows the motor down (Table 3).

Also consistent with the simulation, when the actuators are changed together, an effective cancelation of the motor dynamics happens since the steady-state motor speed remains constant. There is a small dynamic response immediately after the pitch is
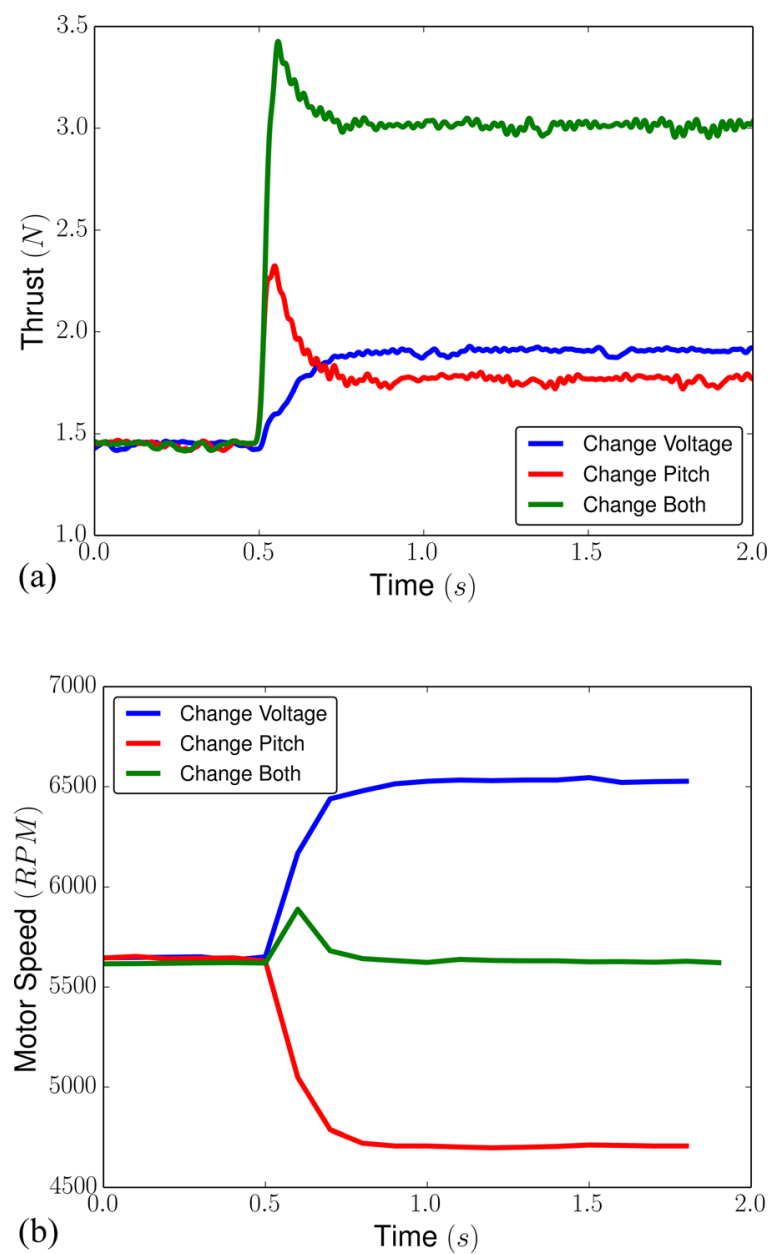

Fig. 6 Experimental thrust and motor speed responses to step increases in motor voltage and propeller pitch. The drop in motor speed after the pitch increase is larger than what was predicted in Fig. 5; however, the shape of the graphs is consistent with the simulated data. When both actuators are used, the motor speed remains essentially constant, showing that the motor dynamics are largely canceled. (a) Experimental thrust response to steps in voltage and pitch and $(b)$ experimental motor speed response to steps in voltage and pitch.
Table 3 Estimated lift and drag coefficients

\begin{tabular}{lccc}
\hline \hline$b_{\mathrm{L}}$ & $b_{\mathrm{D} 1}$ & $b_{\mathrm{D} 2}$ & $b_{\mathrm{D} 3}$ \\
\hline $3.88 \times 10^{-07}$ & $9.96 \times 10^{-09}$ & $2.46 \times 10^{-10}$ & $4.33 \times 10^{-07}$ \\
\hline \hline
\end{tabular}

actuated; however, the motor speed quickly returns to the previous steady-state value. In summary, these experimental results support the theoretical analysis and show that varying the propeller pitch yields fast, almost instantaneous thrust changes.

\section{Trajectory Generation and Control}

The increased thrust rate of change and the availability of negative thrust coming from the addition of variable-pitch propellers are utilized in this section to develop trajectory generation and control algorithms. The control algorithms developed are nonlinear and are not based on near-hover assumptions, allowing for control of aggressive and aerobatic maneuvers. Algorithms generating attitude-specific trajectories that account for actuator saturation levels are also presented. While these algorithms are implemented on a variable-pitch quadrotor, they are general and can be applied to quadrotors with fixed-pitch propellers as well.

4.1 Dynamic Model. Consider the quadrotor helicopter depicted in Fig. 7 with mass, $m$, and mass moment of inertia, $\mathbf{J}$, where $\mathbf{J}$ is aligned with the body $x, y$, and $z$ axes. Let the position of the center of mass of the quadrotor with respect to an inertial frame, $i$, be defined by $\mathbf{r}^{i}$. The attitude of the vehicle in the inertial frame is described by the quaternion $\mathbf{q}$ with the rotational velocities of the vehicle in the body frame, b, being $\boldsymbol{\Omega}^{\mathrm{b}}$. The quaternion convention, $\mathbf{q}=\left[\begin{array}{ll}q^{0} & \mathbf{q}^{T}\end{array}\right]^{\mathrm{T}}$, is used where $q^{0}$ is the scalar portion and $\mathbf{q}$ is the vector portion of the quaternion. In particular, the quaternion rotation operation that rotates the vector $\mathbf{v}$ in $\mathbb{R}^{3}$ from the body frame to the inertial frame is defined as

$$
\left[\begin{array}{c}
0 \\
\mathbf{v}^{i}
\end{array}\right]=\mathbf{q} \otimes\left[\begin{array}{c}
0 \\
\mathbf{v}^{\mathbf{b}}
\end{array}\right] \otimes \mathbf{q}^{*}
$$

where $\mathbf{q}^{*}$ is the quaternion conjugate of $\mathbf{q}$, and $\otimes$ is the quaternion multiplication operator [33]. The inertial-frame time derivative of $\mathbf{q}$ is related to the body rotational velocities by $\dot{\mathbf{q}}=\frac{1}{2} \mathbf{q} \otimes\left[\begin{array}{l}0 \\ \mathbf{\Omega}\end{array}\right]$. Using this quaternion formulation, the Newton-Euler equations of motion that describe the dynamic motion of the quadrotor are given by

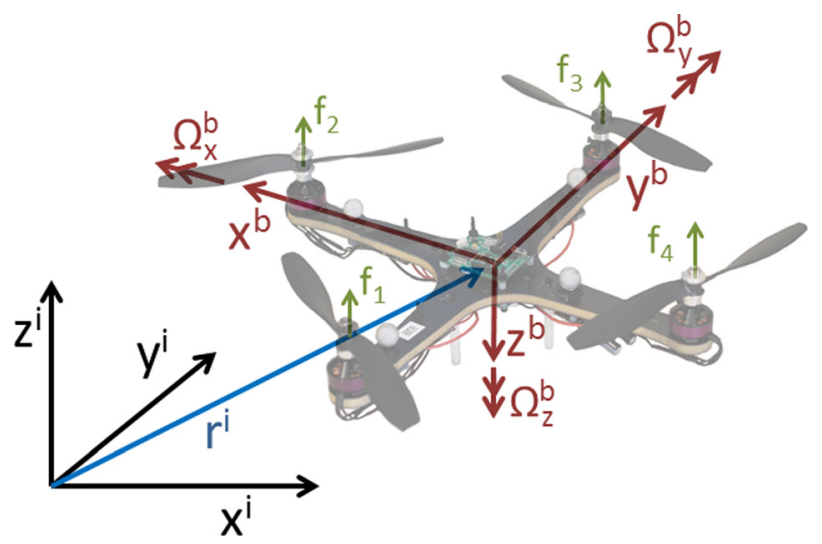

Fig. 7 Quadrotor model and reference frames. Superscript i denotes the inertial frame and superscript $b$ denotes the body frame. 


$$
\begin{gathered}
{\left[\begin{array}{c}
0 \\
\ddot{\mathbf{r}}^{i}
\end{array}\right]=\frac{1}{m} \mathbf{q} \otimes\left[\begin{array}{c}
0 \\
\mathbf{F}^{\mathrm{b}}
\end{array}\right] \otimes \mathbf{q}^{*}-\left[\begin{array}{c}
0 \\
\mathbf{g}^{i}
\end{array}\right]} \\
\dot{\mathbf{\Omega}}=\mathbf{J}^{-1}\left[\mathbf{M}^{\mathrm{b}}-\mathbf{\Omega} \times \mathbf{J} \boldsymbol{\Omega}\right]
\end{gathered}
$$

where $\mathbf{g}^{i}=[0,0, g]^{\mathrm{T}}$ is the inertial-frame gravity vector, $\mathbf{F}^{\mathrm{b}}=[0$, $\left.0, f_{\text {total }}\right]^{\mathrm{T}}$ is the body frame thrust vector, and $\mathbf{M}^{\mathrm{b}}$ is the bodyframe moment vector. The placement of the motors on the quadrotor restricts the generated thrust vector to always be aligned with the body frame $z$-axis.

Let the thrust produced by each of the four motors on the quadrotor be $f_{i}$. The total thrust $f_{\text {total }}$ and quadrotor moments are approximately related to the thrust of each of the four motors by [34]

$$
\left[\begin{array}{c}
f_{\text {total }} \\
\mathbf{M}^{\mathrm{b}}
\end{array}\right]=\left[\begin{array}{cccc}
1 & 1 & 1 & 1 \\
d & 0 & -d & 0 \\
0 & d & 0 & -d \\
-\operatorname{sgn}\left(f_{1}\right) c & \operatorname{sgn}\left(f_{2}\right) c & -\operatorname{sgn}\left(f_{3}\right) c & \operatorname{sgn}\left(f_{4}\right) c
\end{array}\right]\left[\begin{array}{c}
f_{1} \\
f_{2} \\
f_{3} \\
f_{4}
\end{array}\right]
$$

where $d$ is the distance from the center of mass of the vehicle to the motor mount, $c$ is the drag coefficient that relates the yawing moment about the body $z$-axis to the thrust of the four motors, and $\operatorname{sgn}()$ is the signum function. The thrust produced by each motor is bounded between maximum and minimum values as

$$
f_{\min } \leq f_{i} \leq f_{\max }, \quad i=1, \ldots, 4
$$

where $f_{\min }$ and $f_{\max }$ are determined by the physical characteristics of the motor, the available power, the propeller, etc. With fixedpitch propellers, the theoretical minimum thrust is $f_{\min }=0$, but in practice one typically finds that $f_{\min }>0[35,36]$. For a variablepitch system, one can design $f_{\min }=-f_{\max }$.

4.2 Closed-Loop Control. The control algorithms presented in this section utilize a similar trajectory feedback formulation to work presented earlier [7,11]. However, the desired attitude calculation and attitude control are computed using quaternions instead of rotation matrices. The quaternion formulation provides a computationally simple method for calculating attitude errors and control on-board the quadrotor.

Quadrotors are underactuated and differentially flat [11]. The four motor thrust commands can therefore be determined by four flat outputs (and their derivatives): an inertial-frame position reference command, $\mathbf{r}_{\mathrm{d}}^{i}(t)$, in $\mathbb{R}^{3}$ and a desired yaw angle, $\psi_{\mathrm{d}}(t)$. Given the flat outputs, the commanded thrust and moments are computed as follows.

A feedback acceleration vector (the time dependence has been omitted for clarity), $\ddot{\mathbf{r}}_{\mathrm{fb}}^{i}$, is computed as

$$
\ddot{\mathbf{r}}_{\mathrm{fb}}^{i}=-\mathbf{k}_{\mathrm{p}} \mathbf{e}-\mathbf{k}_{i} \int_{0}^{t} \mathbf{e}(\tau) d \tau-\mathbf{k}_{\mathrm{d}} \dot{\mathbf{e}}
$$

where $\mathbf{k}_{\mathrm{p}}, \mathbf{k}_{i}$, and $\mathbf{k}_{\mathrm{d}}$ are positive definite, diagonal, $3 \times 3$ gain matrices, and the error terms are defined as

$$
\begin{aligned}
& \mathbf{e}=\mathbf{r}^{i}-\mathbf{r}_{\mathrm{d}}^{i} \\
& \dot{\mathbf{e}}=\dot{\mathbf{r}}^{i}-\dot{\mathbf{r}}_{\mathrm{d}}^{i}
\end{aligned}
$$

The feedback acceleration vector supplements the commanded (feedforward) acceleration by compensating for errors in position and velocity.

Let the total commanded inertial-frame force required to keep the quadrotor on the desired trajectory be

$$
\mathbf{F}^{i}=m\left(\ddot{\mathbf{r}}_{\mathrm{d}}^{i}+\ddot{\mathbf{r}}_{\mathrm{fb}}^{i}+\mathbf{g}^{i}\right)
$$

This commanded force is used to compute the desired vehicle attitude and the total quadrotor thrust. Rearranging Eq. (13)

$$
m\left(\left[\begin{array}{c}
0 \\
\ddot{\mathbf{r}}^{i}
\end{array}\right]+\left[\begin{array}{c}
0 \\
\mathbf{g}^{i}
\end{array}\right]\right)=\mathbf{q} \otimes\left[\begin{array}{c}
0 \\
\mathbf{F}^{\mathrm{b}}
\end{array}\right] \otimes \mathbf{q}^{*}
$$

Substituting Eq. (20) for the left-hand side of Eq. (21) and normalizing both sides give

$$
\left[\begin{array}{c}
0 \\
\overline{\mathbf{F}}^{i}
\end{array}\right]=\tilde{\mathbf{q}}_{\mathrm{d}} \otimes\left[\begin{array}{c}
0 \\
\overline{\mathbf{F}}^{\mathrm{b}}
\end{array}\right] \otimes \tilde{\mathbf{q}}_{\mathrm{d}}^{*}
$$

where the unit vectors are defined as

$$
\begin{gathered}
\overline{\mathbf{F}}^{i}=\frac{\mathbf{F}^{i}}{\left\|\mathbf{F}^{i}\right\|} \\
\overline{\mathbf{F}}^{\mathrm{b}}=\frac{\mathbf{F}^{\mathrm{b}}}{\left\|\mathbf{F}^{\mathrm{b}}\right\|}=\left[\begin{array}{lll}
0 & 0 & \pm 1
\end{array}\right]^{\mathrm{T}}
\end{gathered}
$$

and $\tilde{\mathbf{q}}_{\mathrm{d}}$ is the desired quadrotor attitude (without accounting for the desired yaw angle) that aligns the body frame thrust vector with the desired inertial-frame force vector. The minimum-angle quaternion rotation between the two unit vectors $\overline{\mathbf{F}}^{i}$ and $\overline{\mathbf{F}}^{\mathrm{b}}$ in $\mathbb{R}^{3}$ is Ref. [37]

$$
\tilde{\boldsymbol{q}}_{\mathrm{d}}=\frac{1}{\sqrt{2\left(1+\overline{\mathbf{F}}^{\mathrm{b}^{\mathrm{T}}} \overline{\mathbf{F}}^{i}\right)}}\left[\begin{array}{c}
1+\overline{\mathbf{F}}^{\mathrm{b}^{\mathrm{T}}} \overline{\mathbf{F}}^{i} \\
\overline{\mathbf{F}}^{\mathrm{b}} \times \overline{\mathbf{F}}^{i}
\end{array}\right]
$$

The sign of the $z$-component of $\overline{\mathbf{F}}^{\mathrm{b}}$ in Eq. (24) is selected so that $\overline{\mathbf{F}}^{\mathrm{b}^{\mathrm{T}}} \overline{\mathbf{F}}^{i} \geq 0$, ensuring that the direction of the body frame thrust vector is aligned with the direction of the inertial-frame acceleration vector.

To fully define the desired attitude of the vehicle, two ambiguities must be addressed. First, quaternions double cover the special orthogonal group $\mathrm{SO}(3)$, meaning $\mathbf{q}$ and $-\mathbf{q}$ represent the same attitude [38]. In practice, this ambiguity is easily addressed by choosing the sign of $\tilde{\mathbf{q}}_{\mathrm{d}}$ at the current time step to agree with the attitude commanded at the previous time step, such that $\tilde{\mathbf{q}}_{\mathrm{d}}^{\mathrm{T}}\left(t_{k}\right) \tilde{\mathbf{q}}_{\mathrm{d}}\left(t_{k-1}\right) \geq 0$. Second, assuming the quadrotor is capable of producing negative thrust, an ambiguity exists between upright and inverted flight because the commanded global acceleration vector is the same in both cases. To fully disambiguate the desired attitude, an additional upright/inverted binary command variable, $\sigma_{\mathrm{d}}(t)= \pm 1$, is needed, where 1 represents upright flight and -1 is inverted.

Finally, the desired vehicle attitude, $\mathbf{q}_{\mathrm{d}}$, is computed by rotating $\tilde{\mathbf{q}}_{\mathrm{d}}$ by the desired yaw angle, $\psi_{\mathrm{d}}$, as

$$
\mathbf{q}_{\mathrm{d}}=\tilde{\mathbf{q}}_{\mathrm{d}} \otimes\left[\cos \left(\psi_{\mathrm{d}} / 2\right) 00 \sin \left(\psi_{\mathrm{d}} / 2\right)\right]^{\mathrm{T}}
$$

The total quadrotor thrust, $f_{\text {total }}$, is computed as $f_{\text {total }}=\left\|\mathbf{F}^{i}\right\|$ since $\left\|\mathbf{F}^{\mathrm{b}}\right\|=\left\|\mathbf{F}^{i}\right\|$ from Eq. (21).

The desired quadrotor attitude rate is found by taking the time derivative of $\overline{\mathbf{F}}$ in the inertial frame. Utilizing the transport theorem [39], this derivative is

$$
\begin{gathered}
\frac{d}{d t} \overline{\mathbf{F}}=\frac{d}{d t}(\overline{\mathbf{F}})_{\mathrm{rel}}+\boldsymbol{\Omega}_{\mathrm{d}} \times \overline{\mathbf{F}} \\
\dot{\overline{\mathbf{F}}}=\Omega_{\mathrm{d}} \times \overline{\mathbf{F}}
\end{gathered}
$$

The first term on the right-hand side of Eq. (27) is zero since $\overline{\mathbf{F}}$ is constant in the body frame. Rearranging Eq. (28) and expressing 
the vectors in the inertial frame give the desired angular rate vector projected onto the $x-y$ plane

$$
\Omega_{\mathrm{d}_{x y}}=\overline{\mathbf{F}}^{i} \times \dot{\overline{\mathbf{F}}}^{i}
$$

The third component of the angular velocity, the yaw rate, is directly computed from the input yaw command as

$$
\Omega_{\mathrm{d}_{z}}=\dot{\psi}_{\mathrm{d}}
$$

The time derivative of $\overline{\mathbf{F}}^{i}$ is explicitly calculated using the quotient rule on Eq. (23) as

$$
\dot{\overline{\mathbf{F}}}^{i}=\frac{\dot{\mathbf{F}}^{i}}{\left\|\mathbf{F}^{i}\right\|}-\frac{\mathbf{F}^{i}\left(\mathbf{F}^{i} \dot{\mathbf{F}}^{i}\right)}{\left\|\mathbf{F}^{i}\right\|^{3}}
$$

where $\dot{\mathbf{F}}^{i}=m\left(\dddot{\mathbf{r}}_{\mathrm{d}}^{i}+\dddot{\mathbf{r}}_{\mathrm{fb}}^{i}\right)$. In practice, $\dddot{\mathbf{r}}_{\mathrm{fb}}^{i}$ is found by numerical differentiating $\ddot{\mathbf{r}}_{\mathrm{fb}}^{i}$.

The calculations of desired attitude and attitude rate assume that $\left\|\mathbf{F}^{i}\right\|=\left\|\mathbf{F}^{\mathrm{b}}\right\| \neq 0$, stemming from the fact that the attitude of the vehicle is irrelevant to the motion of the center of mass of the vehicle during free-fall. However, the vehicle attitude becomes important the moment the vehicle exits free-fall and so should be controlled the entire time. In practice, this attitude ambiguity is accounted for by ensuring that the reference trajectory does not command free-fall for a nontrivial amount of time. New desired attitude and attitude rates are computed only when $\left\|\mathbf{F}^{i}\right\|$ is above a small threshold, maintaining the previously commanded attitude and attitude rates while $\left\|\mathbf{F}^{i}\right\|$ is close to zero.

Utilizing the sequential rotation properties of quaternions [33], the desired vehicle attitude can be represented as a rotation from the inertial frame to the actual frame of the vehicle followed by a rotation from the vehicle frame to the desired vehicle orientation, as in

$$
\underbrace{\mathbf{q}_{\mathrm{d}}}_{\text {inertial frame }}=\underbrace{\mathbf{q}}_{\text {inertial frame }} \otimes \underbrace{\mathbf{q}_{\mathrm{e}}}_{\text {body frame }}
$$

The quaternion $\mathbf{q}_{\mathrm{e}}$ represents the error quaternion, or the attitude error of the vehicle expressed in the body frame. Rearranging Eq. (32) using the conjugate properties of the quaternion yields the error quaternion, expressed in the body frame, as a simple quaternion multiplication between the actual attitude and the desired attitude



Equations (32) and (33) are similar to equations in previous work [40]; however, in this paper, the order of the quaternion multiplication differs so as to agree with standard notation and the rotation operation introduced in Eq. (12) [33].

With the error quaternion expressed in the body frame, the elements of the quaternion directly map to the required body frame moments. Similar to other quaternion-based attitude control laws proposed [41-43], the attitude control is accomplished using proportional derivative control on the attitude error and attitude rate error as

$$
\mathbf{M}^{\mathrm{b}}=\operatorname{sgn}\left(q_{\mathrm{e}}^{0}\right) \mathbf{K}_{\mathrm{p}} \mathbf{q}_{\mathrm{e}}-\mathbf{K}_{\mathrm{d}}\left(\Omega^{\mathrm{b}}-\Omega_{\mathrm{d}}^{\mathrm{b}}\right)
$$

where $q_{\mathrm{e}}^{0}$ and $\mathbf{q}_{\mathrm{e}}$ are the scalar and vector portions of the error quaternion, respectively. The gain matrices, $\mathbf{K}_{\mathrm{p}}$ and $\mathbf{K}_{\mathrm{d}}$ are diagonal and positive definite. The diagonal elements of the gain matrices are hand tuned on the real variable-pitch quadrotor.
Finally, given $f_{\text {total }}$ and $\mathbf{M}^{\mathrm{b}}$, the closed-loop control is completed by calculating the corresponding motor thrust commands through inverting the relationship in Eq. (15). Note that Eq. (15) cannot be inverted directly as it depends on the sign of the unknown motor thrust values. Thus, in practice, we use the sign of $f_{\text {total }}$ as an approximation for the $\operatorname{sgn}\left(f_{i}\right)$ functions. Part of the reason for using this approximate solution rather than computing the true solution to the nonlinear equation is that the attitude loop is run on a 16-bit microcontroller at $1 \mathrm{kHz}$, and so the computational resources and time available to solve Eq. (15) are severely limited. The motor thrust commands are translated into propeller pitch and motor speed commands using the control allocation policy introduced in Sec. 3.3.

4.3 Trajectory Generation. Given the control structure capable of tracking position and yaw reference commands developed in Sec. 4.2, consider the problem of navigating through $n$ waypoints in three-space. Similar to previous work [11,34], a trajectory consisting of piecewise smooth polynomials of order $m$ over $n-1$ time intervals is used. The proposed algorithm extends the existing literature by generating trajectories based on the physical limitations of the hardware actuators. This algorithm finds time-optimal polynomial paths subject to vehicle actuator saturation, allowing for paths tailored to the actuators of a specific vehicle. Additionally, a method for embedding attitude-specific constraints along the reference path is developed, allowing for aerobatic maneuvers such as flips to be performed with a single control law.

The trajectory of the quadrotor is defined by

$$
\mathbf{r}_{\mathrm{d}}^{i}(t)=\left\{\begin{array}{cc}
\sum_{i=0}^{m} \alpha_{i, 1} t^{i} & 0 \leq t<t_{1} \\
\sum_{i=0}^{m} \alpha_{i, 2} t^{i} & t_{1} \leq t<t_{2} \\
\vdots & \vdots \\
\sum_{i=0}^{m} \alpha_{i, n-1} t^{i} & t_{n-2} \leq t \leq t_{n-1}
\end{array}\right.
$$

where $\alpha_{i, n}$ is the $i$ th polynomial coefficient over the $n$th time interval. In addition to the advantages of polynomial-based trajectories noted in existing literature [11], the proposed algorithm utilizes the fact that, given the correct number of endpoint constraints at the segment boundaries and the corresponding segment times, a closed-form solution for finding the polynomial coefficients exists.

As an example, consider the $x$-dimension of a two waypoint problem, where the vehicle starts and stops in hover. As described in Sec. 4.2, the inputs to the quadrotor are computed as a function of the first three derivatives of the position command. To ensure that those inputs are smooth, the initial and final first four derivatives of position are constrained as

$$
\begin{gathered}
\mathbf{r}_{\mathrm{d}_{x}}^{i}(0)=x_{0} \quad \mathbf{r}_{\mathrm{d}_{x}}^{i}\left(t_{\mathrm{f}}\right)=x_{\mathrm{f}} \\
\mathbf{r}_{\mathrm{d}_{x}}^{i^{(k)}}(0)=0 \quad \mathbf{r}_{\mathrm{d}_{x}}^{i^{(k)}}\left(t_{\mathrm{f}}\right)=0 \quad k=1, \ldots, 4
\end{gathered}
$$

where the superscript in parentheses represents the $k$ th time derivative of $x$. The formulation results in ten constraints, five initial, and five terminal conditions. Therefore, assuming the final time, $t_{\mathrm{f}}$, is known, a nine-order polynomial offers a closed-form solution to the problem.

Next, consider the same initial and final conditions, but now with $n-2$ intermediate waypoints that the trajectory must pass through. Assuming a desired arrival time associated with each waypoint is known, the problem maintains a closed-form solution as long as there are $10 n-10$ constraints. Constraining the position and first four derivatives of position at each waypoint provides the 
required number of constraints; however, this requires knowledge of the velocity, acceleration, jerk, and snap of the quadrotor at each waypoint. Alternatively, if only the position of the waypoint is important, the remaining $8(n-2)$ constraints are formed by ensuring continuity of the first eight derivatives of position at the $n-2$ intermediate waypoints.

Example boundary conditions with waypoints $\mathbf{w}=\left[w_{0}, w_{1}, \ldots\right.$, $\left.w_{n-1}\right]$ are given by

$$
\begin{aligned}
& \text { initial }= \begin{cases}\mathbf{r}_{d}^{i}(0)=w_{0} & k=1, \ldots, 4 \\
\mathbf{r}_{\mathrm{d}}^{i^{(k)}}(0)=0 & r=1, \ldots, n-2\end{cases} \\
& \text { middle }= \begin{cases}\mathbf{r}_{\mathrm{d}}^{i}\left(t_{r}^{+}\right)=w_{r} & r=1, \ldots, n-2 \\
\mathbf{r}_{\mathrm{d}}^{i}\left(t_{r}^{-}\right)=w_{r} & k=1, \ldots, 8 \\
\mathbf{r}_{\mathrm{d}}^{i^{(k)}}\left(t_{r}^{+}\right)-\mathbf{r}_{\mathrm{d}}^{i^{(k)}}\left(t_{r}^{-}\right)=0\end{cases} \\
& \text { final }= \begin{cases}\mathbf{r}_{\mathrm{d}}^{i}\left(t_{n-1}\right)=w_{n-1} \\
\mathbf{r}_{\mathrm{d}}^{i^{(k)}}\left(t_{n-1}\right)=0\end{cases}
\end{aligned}
$$

As the constraints are formulated, the path starts and ends at hover and is required to pass through each of the waypoints.

Note that the formulation offers flexibility by allowing any of the first four derivatives of position to be user-specified at any of the intermediate waypoints. For instance, if the desired $x$ component of velocity at waypoint $j$ is $v_{j}$, the constraint becomes $\mathbf{r}_{\mathrm{d}_{x}}^{i}\left(t_{j}^{-}\right)^{(1)}=\mathbf{r}_{\mathrm{d}_{x}}^{i}\left(t_{j}^{+}\right)^{(1)}=v_{j}$. When the velocity is not specified, the constraint is $\mathbf{r}_{\mathrm{d}_{x}}^{i}\left(t_{j}^{-}\right)^{(1)}-\mathbf{r}_{\mathrm{d}_{x}}^{i}\left(t_{j}^{+}\right)^{(1)}=0$. Constraining any of the derivatives of an intermediate waypoint to a known value is accomplished by removing two of the higher-order continuity constraints at that waypoint. As long as the waypoint time and the initial and final conditions are specified, the solution for the desired trajectory and all its derivatives is closed-form and consists of a single matrix inversion. This is a departure from previous work where polynomial coefficients were found by solving a quadratic program [11]. Care must be taken, however, when specifying several constraints at a single node of the polynomial. Position, its derivatives, and time are highly coupled and radical solutions to the polynomial formulation can be found when the constraints are not chosen properly. Section 4.4 proposes a method for ensuring the resulting paths are within the limits of the actuators of the vehicle.

4.4 Actuator-Constrained Minimum-Time Polynomial Trajectory Generation. While the preceding closed-form polynomial trajectory generation method ensures that all the reference commands to the quadrotor will be smooth, there is no guarantee that the commands will be within the feasible limits of the hardware actuators. For instance, any trajectory of nonzero length will become infeasible as the segment times approach zero because the corresponding velocity, acceleration, and attitude rate reference commands will approach infinity. This section presents a novel optimization method for finding the minimum segment times subject to the physical constraints of the quadrotor.

Given the waypoints and path constraints from Sec. 4.3, the optimization returns the segment times that minimize the total path time subject to the motor saturation constraints in Eq. (16). The optimization over $n$ waypoints with $\mathbf{t}=\left[\begin{array}{llll}t_{1} & t_{2} & \ldots & t_{n-1}\end{array}\right]$ segment times is formulated as

$$
\begin{gathered}
\quad \mathbf{t}=\underset{\mathrm{t}}{\operatorname{argmin}} \quad t_{n-1} \\
\text { subject to } \quad f_{\min } \leq f_{i} \leq f_{\max } \quad i=1, \ldots, 4 \\
t_{j}>0 \quad j=1,2, \ldots, n-1
\end{gathered}
$$

The trajectory starts at the first waypoint with $t_{0}=0$. The decision variables $\mathbf{t}$ are the times at which the quadrotor passes through the $n-1$ remaining waypoints. Minimizing the last decision variable minimizes the total time of the trajectory since each segment time is constrained to be positive. A path is defined as feasible when none of the motor commands exceeds the allowable motor thrust values. The calculation of these motor constraints is detailed below.

During each iteration of the optimizer, the reference path is calculated by solving the closed-form polynomial formulation for the coefficients $\alpha_{i, n}$ as specified above using the current value of $\mathbf{t}$. The equations of motion of the quadrotor in Eqs. (13) and (14) are then inverted using the computed path as the reference command, returning the required forces and moments to fly that path. The individual motor thrust values are found by inverting the relationship in Eq. (15). The calculated motor thrust values are only an approximation of the true thrust values commanded during flight due to errors in estimated model parameters (mass and inertia) and errors from ignoring the feedback control in Eqs. (17) and (34) (inverting the equations of motion using the reference path as the input assumes the quadrotor never deviates from the reference path).

The resulting segment times found from the optimization do not guarantee that the commanded motor thrusts will never exceed the prescribed bounds; however, in practice $f_{\max }$ and $f_{\min }$ can be treated as tuning gains. Decreasing the allowable thrust window for each motor decreases the overall aggressiveness of the resulting paths, providing a way to decrease the potential for actuator saturation.

The constraints in the optimization problem are complicated, nonlinear functions of the decision variables and so convergence to globally optimal segment times cannot theoretically be guaranteed. However, the problem always has a feasible solution because, as the segment times increase, the motor values approach hover conditions, indicating that each desired trajectory has a feasible solution. Also, in our experience, simple interior-point optimization methods work very well on this problem without ever converging to obviously suboptimal local minima.

The optimization problem assumes that no change in the yaw angle of the quadrotor is commanded. Note that the yaw of the vehicle does not depend on the trajectory flown, and so the desired yaw and yaw rate can be specified after the trajectory is found. However, the extra control authority needed to actuate the yaw must be accounted for in the optimization by setting $f_{\max }$ and $f_{\min }$ appropriately. As the vehicle is symmetric about yaw, for the experiments in this paper, we left the yaw constant throughout the trajectories flown.

4.5 Attitude Constraints. In addition to accounting for actuator saturation, the proposed method allows for specific attitude constraints to be incorporated into the desired path formulation by constraining the acceleration of the vehicle based on Eq. (21). Given a desired inertial-frame attitude $\mathbf{q}_{\text {des }}$, the corresponding required inertial-frame acceleration $\ddot{\mathbf{r}}_{\text {att }}^{i}$ is computed, up to an overall scale factor of the thrust magnitude, by solving

$$
\left[\begin{array}{c}
0 \\
\ddot{\mathbf{r}}_{\text {att }}^{i}
\end{array}\right]=\frac{\left\|\mathbf{F}^{b}\right\|}{m} \mathbf{q}_{\text {des }}^{*}\left[\begin{array}{l}
0 \\
0 \\
0 \\
1
\end{array}\right] \mathbf{q}_{\text {des }}-\left[\begin{array}{c}
0 \\
\mathbf{g}^{i}
\end{array}\right]
$$

where $\left\|\mathbf{F}^{\mathrm{b}}\right\|$ is chosen to scale the acceleration as desired. Equation (40) allows the user to specify the attitude of the vehicle at polynomial nodes in the path. While the vehicle attitude between nodes is not directly specifiable with the current algorithm, designating the vehicle attitude at a certain point in space can be beneficial for maneuvers such as flying through windows or performing aerobatics. 
4.6 Trajectory Generation Simulations. Figure 8 shows an example path found with the optimization scheme in Sec. 4.4. The path is constrained to start and stop in hover and to pass through three intermediate waypoints, indicated in the plot by red stars. Initially, the time between each waypoint is arbitrarily set to be $0.55 \mathrm{~s}$. This yields a strangely oscillatory path with motor commands that saturate significantly. After running the optimization routine, the motor commands are within the saturation bounds and the resulting path avoids the oscillations from the initial path. The aggressiveness of the path is easily tunable by lowering the saturation bounds of the actuators at the expense of an increased path flight time.

Figure 9 shows an example trajectory that illustrates a potential benefit of variable-pitch over fixed-pitch propellers for a quadrotor. The trajectory starts at the origin at hover and ends at hover $1 \mathrm{~m}$ higher. The negative thrust range of the variable-pitch propellers allows the quadrotor to quickly generate upward thrust to enable fast deceleration. The fixed-pitch quadrotor, however, relies on gravity to decelerate and so requires a longer overall trajectory time than the variable-pitch quadrotor.

As discussed in Sec. 4.2, the attitude is not well defined from Eq. (25) when $\left\|\mathbf{F}^{\mathrm{b}}\right\|=0$ (the vehicle is in free-fall). However, interesting attitude maneuvers can be constructed by imposing an instantaneous free-fall constraint. In particular, Figure 10 shows
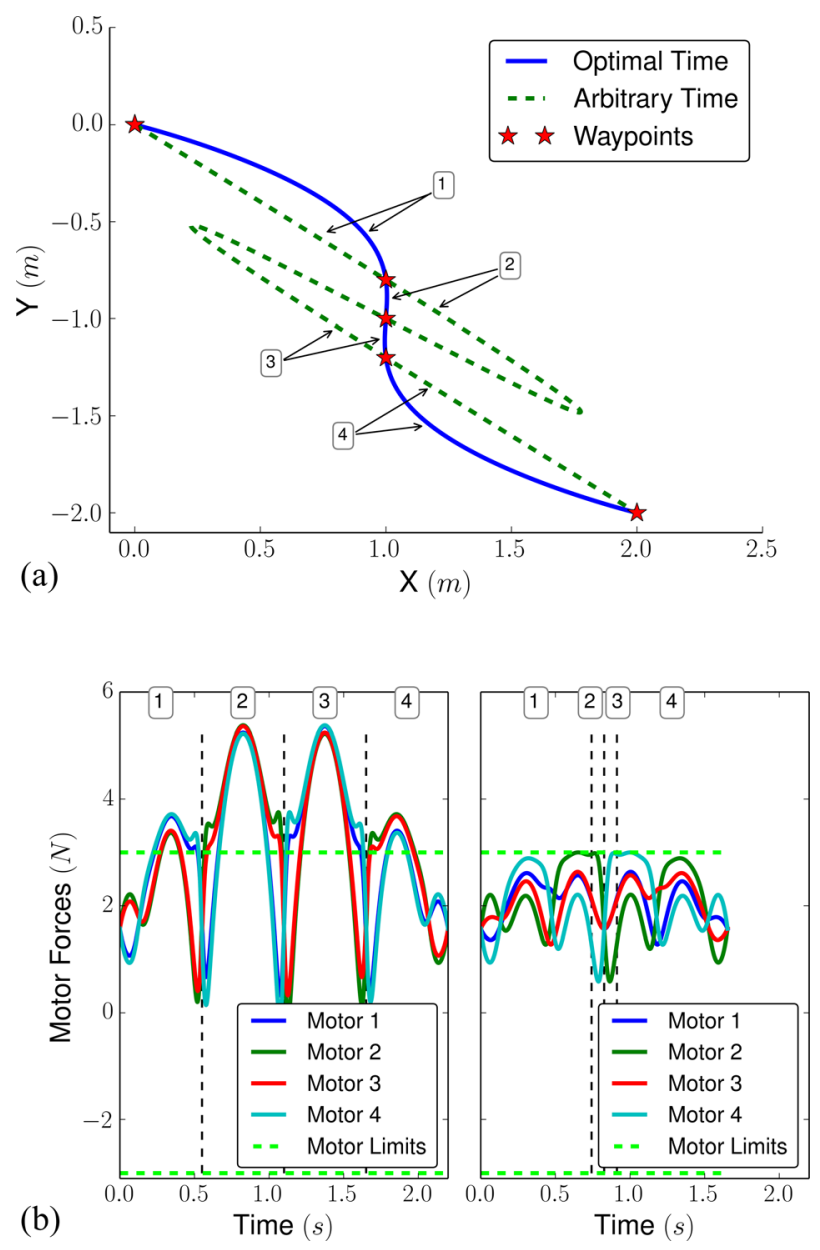

Fig. 8 Example path showing the minimum-time optimization. Both paths satisfy the constraints of starting and ending at hover and passing through the five waypoints; however, the optimal time path keeps the motor commands from saturating and completes the path in less time than the one with arbitrary waypoint arrival times. Each path segment is numbered, with the vertical dashed lines showing the time allotted for each segment. (a) Example path and (b) commanded motor values. the trajectory generated by imposing an acceleration constraint of $-\mathbf{g}^{i}$ between two hover conditions at different locations along the $x$-axis. The quadrotor goes inverted after the instantaneous freefall because $\sigma(t)$ is changed from 1 to -1 at that point.

Attitude constraints embedded in the path formulation are utilized to command a path similar to the backflip demonstrated on the Stanford STARMAC quadrotor [8]. Simulation results of the path are presented in Fig. 11. The flipping motion is prescribed by embedding a -90 deg roll constraint just before the apex of the path and a 90 deg roll constraint just after the apex.

\section{Hardware and Software Implementation}

The variable-pitch quadrotor used in this project was designed and built at MIT's ACL using mostly off-the-shelf components. A closeup of the vehicle is shown in Fig. 12. The frame is cut from a sheet of carbon fiber sandwich material with a balsa wood core. The square shape is designed to minimize vibrations induced by the propellers, motors, and servos because the vibrations cause the attitude estimate by the on-board sensors to quickly deteriorate.

The pitch control mechanisms are commercial parts designed for small remote-controlled airplanes (see Fig. 13(a)). They use a carbon fiber pushrod routed through the center of a hollow motor shaft. A servo is mounted beneath the motor to actuate the propeller pitch. Note that swashplates needed on single-rotor helicopters to achieve agile flight (an example, swashplate on a remotecontrolled helicopter is shown in Fig. 13(b)) are mechanically much more complicated than the variable-pitch mechanism used on the quadrotor in this project.

Other than slightly increasing the overall vibration in the vehicle and potentially decreasing the propeller efficiency by requiring symmetric blades, the addition of variable-pitch propellers to the quadrotor has few adverse affects when compared to the mechanical simplicity of traditional fixed-pitch quadrotors. As shown in Table 4 , the servos and variable-pitch components only make-up about $13 \%$ of the total weight of the vehicle, with the vehicle maintaining at least a 2:1 thrust-to-weight ratio including these additional components.

An overview of the software and data flow is shown in Fig. 14. For the indoor flight results presented in this paper, the vehicle state estimation is aided by an external motion capture system. For outdoor flight, the vehicle state could be estimated using a standard inertial measurement unit and global positioning system

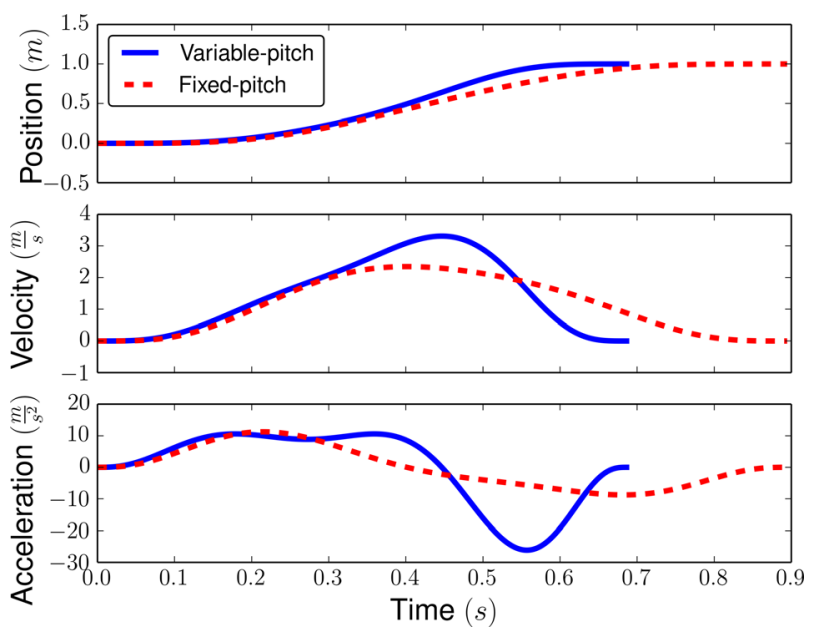

Fig. 9 Two example vertical flight trajectories computed using the optimization routine in Sec. 4.4. Both trajectories have the same upper bound on motor thrust. The variable-pitch trajectory has a negative thrust lower bound, but the fixed-pitch trajectory has a lower bound of near zero. Note that the variable-pitch trajectory is shorter because it decelerates by quickly generating upward thrust. 

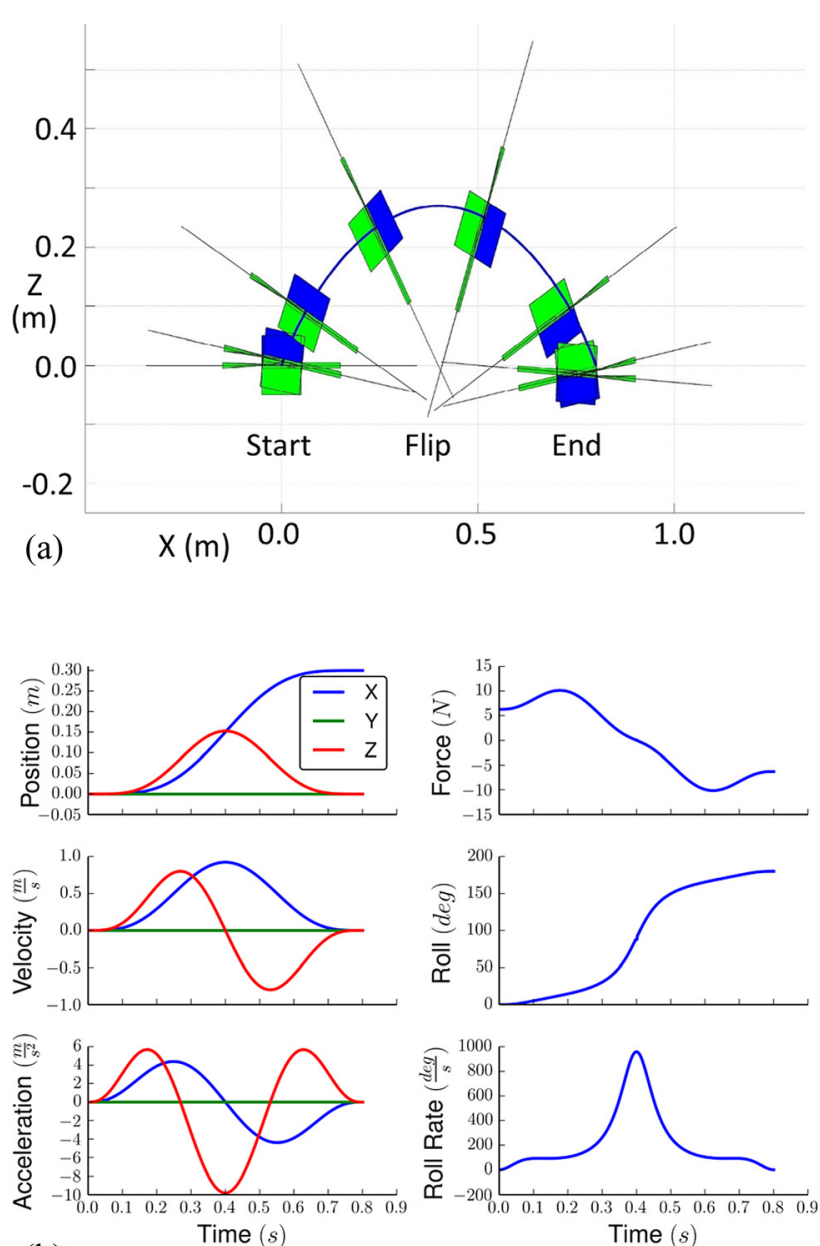

(b)

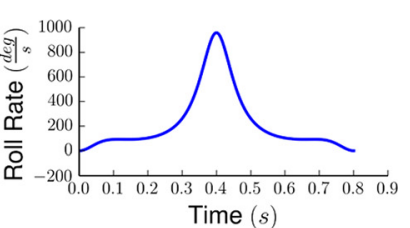

Fig. 10 Trajectory generated by imposing a position-free freefall acceleration condition between two hover waypoints along the $x$-axis. The small corner in the commanded attitude trajectory comes from not computing new commanded attitudes when the total force command is close to zero. The vehicle goes inverted at the apex of the trajectory by explicitly changing $\sigma(t)$ from 1 to -1 . (a) Flip trajectory and $(b)$ commanded state trajectory values.

[44]. Position, velocity, and attitude estimates from the motion capture system are available to the control system at $200 \mathrm{~Hz}$. These state estimates are combined with the desired states from the trajectory generation algorithm to form commanded thrust, attitude, and attitude rate values that are sent to the autopilot at

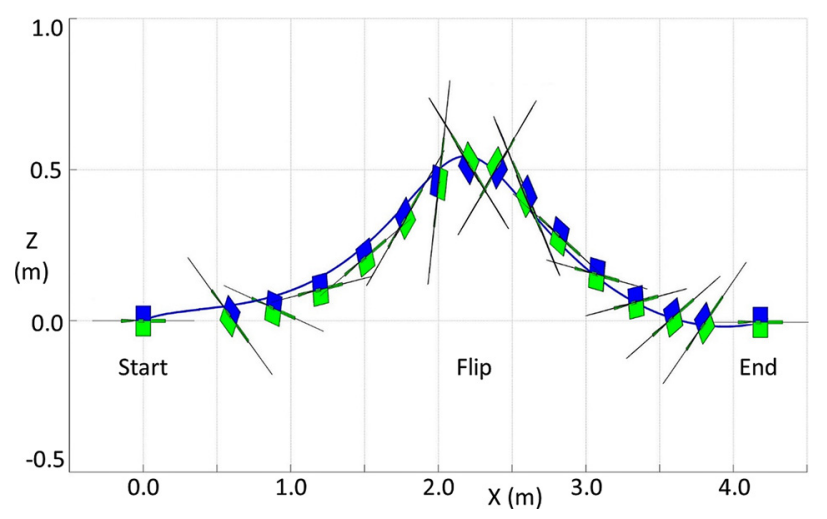

Fig. 11 Simulation results of a 360 deg backflip. The flip is specified using a -90 deg roll constraint before the peak of the trajectory and a 90 deg roll constraint after the peak. The quadrotor starts and ends in hover.

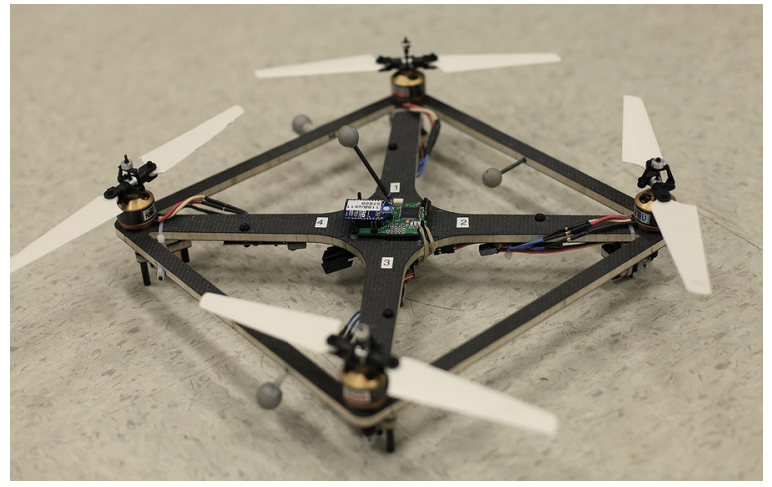

Fig. 12 ACL Variable-pitch quadrotor. The servos that actuate the variable-pitch propellers are visible under each of the motors. The quadrotor frame measures $0.35 \mathrm{~m}$ across.
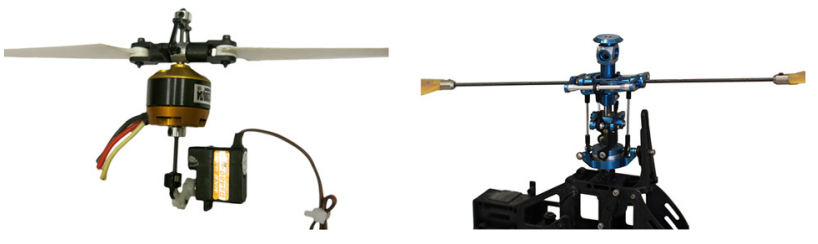

Fig. 13 Left: one of the pitch actuation mechanisms on the current version of the variable-pitch quadrotor. Right: a typical swashplate on a remote-controlled helicopter. (a) Variable-pitch actuator and (b) helicopter swashplate.

$100 \mathrm{~Hz}$ (limited by the bandwidth of the wireless radio). The autopilot then generates motor and pitch commands to control the quadrotor.

The low-level attitude estimation and control are performed on a custom autopilot, nick-named the "UberPilot," developed for this project. The UberPilot circuit design is loosely based on the UAV Development Board version 4 from Sparkfun ${ }^{1}$ and features a $10 \mathrm{~g}$ (14 g with the wireless radio), $1.5 \times 2.0 \mathrm{in}$. autopilot board. The autopilot board plugs into a $20 \mathrm{~g}$, similarly sized power board that distributes power and signal lines from the battery and the autopilot to the four motors and servos. The autopilot and power distribution boards are shown in Fig. 15.

The UberPilot utilizes a 16-bit dsPIC33F microcontroller from Microchip Technologies Inc. ${ }^{2}$ running at $50 \mathrm{MHz}$ and a three-axis rate gyro (ITG-3200) from InvenSense. ${ }^{3}$ Communication from the UberPilot to the ground station is performed using a $2.4 \mathrm{GHz}$ $\mathrm{xBee}$ wireless radio.

Estimates of the vehicle attitude are computed on the UberPilot by integrating the rate gyro measurements. Drift in the gyro measurements and the corresponding degradation of the attitude estimates is accounted for by correcting the on-board attitude estimates with attitude measurements from the motion capture software. These external attitude estimates are sent via the wireless radio to the on-board processor and are added to the internal attitude estimates using a simple complimentary filter as

$$
\mathbf{q}_{\mathrm{o}}[\mathrm{i}+1]=\mathbf{q}_{\mathrm{o}}[\mathrm{i}]+k\left(\mathbf{q}_{\mathrm{e}}[\mathrm{i}+1]-\mathbf{q}_{\mathrm{o}}[\mathrm{i}]\right)
$$

where $q_{o}$ is the on-board quaternion estimate, $q_{e}$ is the external quaternion measurement, $i$ is the discrete time index, and $k$ is a gain such that the time constant of the first-order system is on the order of 5-10 s.

The Uberpilot samples the rate gyros at $1 \mathrm{kHz}$ and computes an attitude solution at the same rate. The motor and servo commands

\footnotetext{
${ }^{1}$ http://www.sparkfun.com

${ }^{2} \mathrm{http}: / /$ www.microchip.com

${ }^{3} \mathrm{http}: / /$ www.invensense.com
} 
Table 4 Overall weight of the various components of the variable-pitch quadrotor

\begin{tabular}{lllll}
\hline \hline Component & \multicolumn{1}{c}{ Type } & Qty & Unit weight (g) & Total weight (g) \\
\hline Frame & Custom & 1 & 90 & 90 \\
Battery & 3 cell 850 mA & 1 & 79 & 79 \\
Motor & Axi 2208/34 EVP & 4 & 45 & 180 \\
ESC & Mikrokopter & 4 & 8.5 & 34 \\
Servo & MKS DS480 & 4 & 10 & 40 \\
Propellers & MS composit EVPU & 4 & 8 & 32 \\
Variable-pitch actuator & MS composit EVPU & 4 & 8 & 32 \\
Autopilot & Custom & 1 & 14 & 14 \\
Power distribution board & Custom & 1 & 20 & 20 \\
Wiring, connectors, etc. & N/A & N/A & 20 & 541 \\
Vehicle weight & & & \\
\hline \hline
\end{tabular}

The servos and variable-pitch actuators make up about 13\% of the overall weight of the quadrotor. The vehicle has well over a 2:1 thrust-to-weight ratio, including these variable-pitch actuators.



Fig. 14 Overview of the software and data flow for the variablepitch quadrotor. The off-board algorithms run on a desktop personal computer. All off-board communication is handled via the robot operating system.

are sent to their respective hardware actuators at $1 \mathrm{kHz}$, although the servo only responds to commands at $333 \mathrm{~Hz}$.

\section{Experimental Results}

Results of the control and trajectory generation techniques developed in Sec. 4 as implemented on the variable-pitch quadrotor are presented in this section. All the flights are performed in the RAVEN flight testing facility at the Massachusetts Institute of Technology $[45,46]$.

In terms of the trajectory generation algorithm presented in Sec. 4.3 , the variable-pitch quadrotor is advantageous because the addition of negative thrust more than doubles the effective thrust range for each of the four motors when compared to an equivalently powered fixed-pitch quadrotor. The reverse thrust capabilities of the variable-pitch quadrotor enable both inverted flight

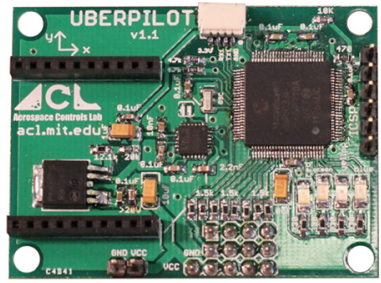

(a)

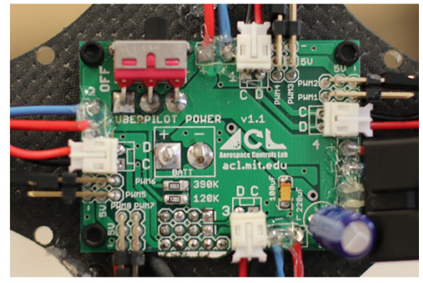

(b)
Fig. 15 Custom electronics used to perform attitude estimation and control on the variable-pitch quadrotor. The control board (left) mounts on top of the quadrotor and houses a 16-bit microcontroller, three-axis rate gyro, and wireless radio. The power distribution board (right) mounts beneath the control board and distributes power from the battery and signal lines to the ESCs and servos. (a) UberPilot control board and (b) UberPilot power distribution board.

Journal of Dynamic Systems, Measurement, and Control and vertical decelerations higher than gravity. As discussed in Sec. 3, variable-pitch propellers also increase the available controller bandwidth by effectively canceling the motor dynamics. The variable-pitch propellers are thus able to change thrust substantially faster than corresponding fixed-pitch propellers.

Flight results in this section demonstrate the ability of the quadrotor to track paths upright and inverted, quickly decelerate, and perform aerobatic maneuvers using the position-based trajectory generation method.

6.1 Inverted and Upright Tracking. The variable-pitch quadrotor utilizes symmetric propellers. Combined with the inherent symmetry of the vehicle design, these propellers permit the quadrotor to fly equally well upright or inverted. The first set of flight results, shown in Fig. 16, demonstrates the ability of the vehicle to track the same path in either the upright or the inverted configuration.

6.2 Negative Thrust Decelerations. One of the primary advantages of the variable-pitch propellers for a quadrotor is the ability to generate negative thrust. While this allows the vehicle to fly upside down, it also brings the capability to decelerate quickly by momentarily reversing the propeller pitch to create upward thrust. This capability is highlighted in Fig. 17. In variable-pitch mode, the quadrotor is able to track the reference position command with only $1 \%$ overshoot, compared to $60 \%$ overshoot when flying in fixed-pitch mode. The improved tracking performance in variable-pitch mode is due primarily to the large negative accelerations that can be achieved when the pitch of the propellers is

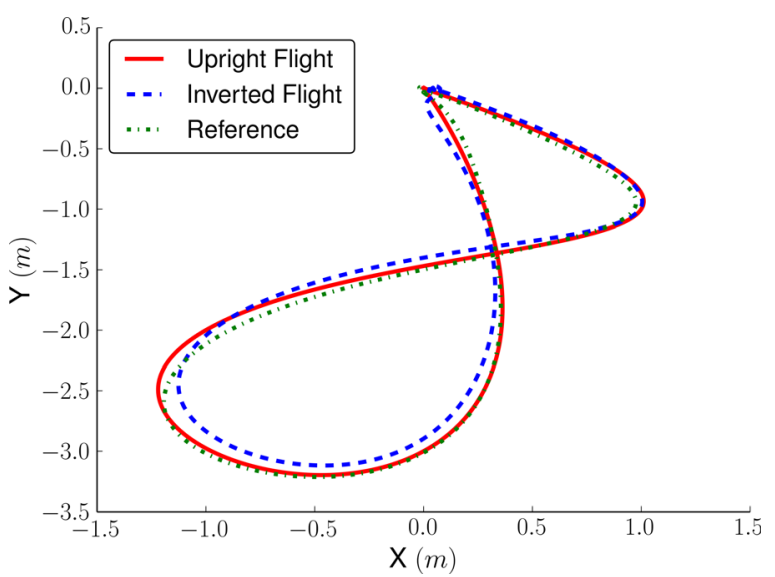

Fig. 16 Path tracking qualities of the quadrotor. The vehicle is commanded to follow the same path both upright and inverted. Symmetry in the vehicle and propellers allows for similar flight characteristics upright or inverted. 

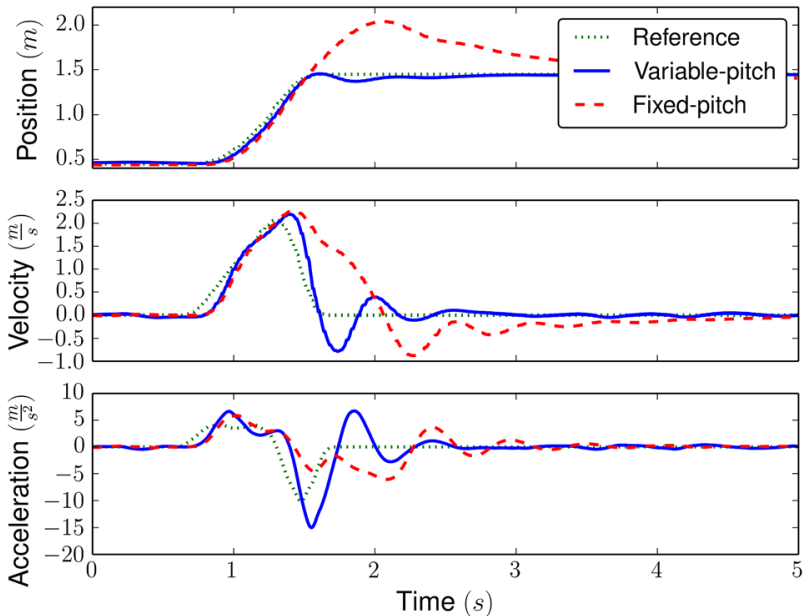

Fig. 17 Flight data for the variable-pitch quadrotor flying the same trajectory in variable-pitch mode and in fixed-pitch mode. The variable-pitch propellers allow for faster decelerations and better tracking of the position reference command.

allowed to vary. Note that the reference command requires changes in acceleration, which are as fast as or faster than the response of the fixed-pitch motor from Fig. 6. This fast change in acceleration is another reason the vehicle is unable to track the reference command well when flying in fixed-pitch mode.

6.3 Flips. As described in Sec. 4.6, interesting aerobatic maneuvers can be performed using the trajectory generation method by embedding attitude constraints along the path. The first example, snapshots of which are shown in Fig. 18, shows the quadrotor performing a $180 \mathrm{deg}$ flip. The quadrotor is commanded to follow a parabolic trajectory in the $x-z$ plane, starting and stopping at hover, with a $\mathbf{- g}$ acceleration constraint imposed in the middle. At the apex of the parabola, the quadrotor is commanded to fly inverted, resulting in a $180 \mathrm{deg}$ flipping maneuver.

Figure 19 shows the angular position and rate tracking abilities of the variable-pitch quadrotor during the $180 \mathrm{deg}$ flip. The entire maneuver takes less than $0.4 \mathrm{~s}$ and the quadrotor rotates at over $1000 \mathrm{deg} / \mathrm{s}$.

Next, a 180 deg flip is embedded into a translating path to demonstrate the ability of the vehicle and algorithms to perform moving aerobatics. This maneuver is shown in Fig. 20. The vehicle starts and stops at hover and travels nearly $4 \mathrm{~m} / \mathrm{s}$ forward and $2 \mathrm{~m} / \mathrm{s}$ upward just before the flip.

Finally, snapshots of hardware results of the STARMAC inspired backflip (simulation results are shown in Fig. 11) are shown in Fig. 21. The backflip is similar to the translating $180 \mathrm{deg}$ flip in Fig. 20 except the quadrotor performs a full 360 deg flip.
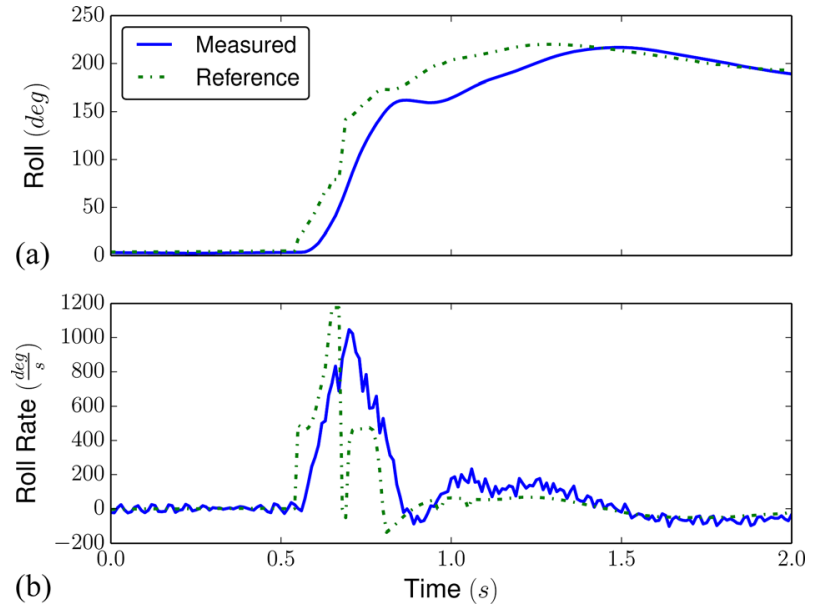

Fig. 19 Commanded and measured roll and roll rate values from the quadrotor following a flipping maneuver. The measured values come from the on-board rate gyros. The flip takes less than $0.4 \mathrm{~s}$ to complete. Snapshots of the quadrotor during the flip are shown in Fig. 18.

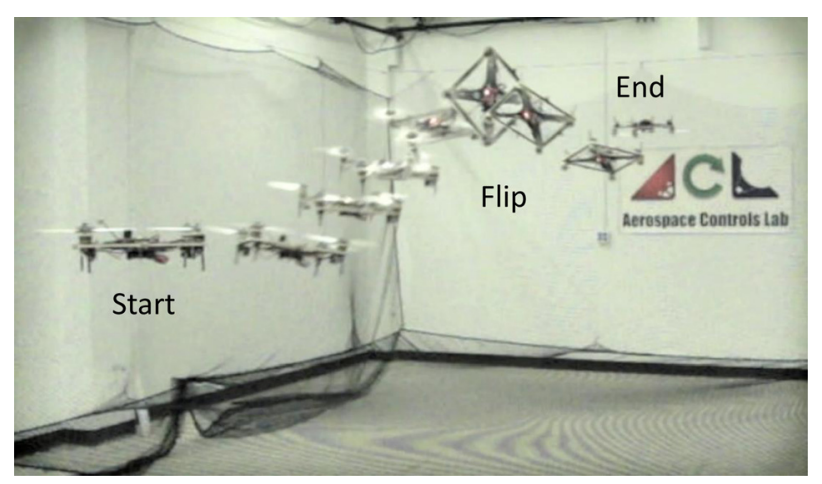

Fig. 20 The quadrotor performing a translating 180 deg flip. The vehicle starts and ends at hover and performs a half back flip in the middle of the path. The vehicle travels forward at nearly $4 \mathrm{~m} / \mathrm{s}$ during the maneuver.

This maneuver is difficult for the quadrotor to execute because it requires a relatively high deceleration after the flip. As the quadrotor exits the flip, the vehicle is moving about $3 \mathrm{~m} / \mathrm{s}$ forward and over $2 \mathrm{~m} / \mathrm{s}$ downward. The vehicle requires nearly full thrust to slow to a stop. The optimization algorithm from Sec. 4.4 is used to find this feasible trajectory, preventing the vehicle from becoming unstable due to actuator saturation.

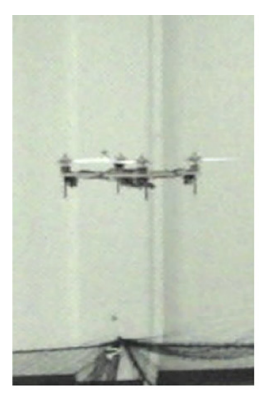

(a)

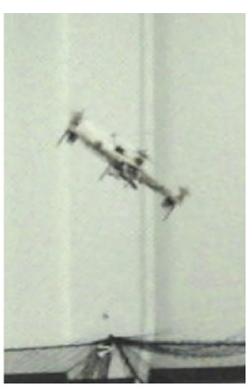

(b)

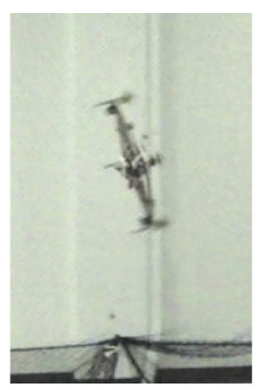

(c)

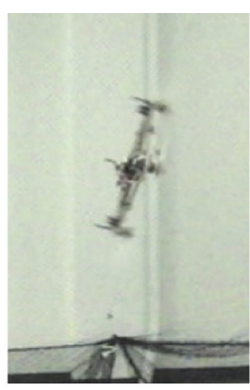

(d)

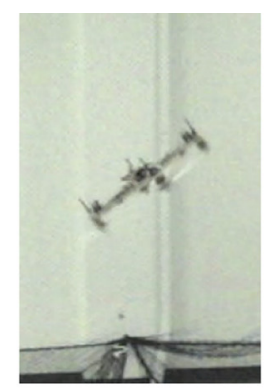

(e)

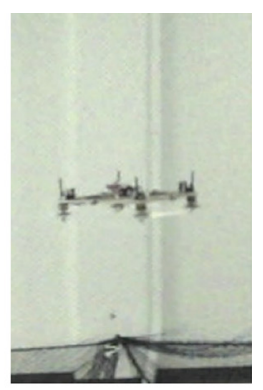

(f)

Fig. 18 Variable-pitch quadrotor performing a $180 \mathrm{deg}$ flip by embedding a 90 deg roll constraint at the top of an arc in the $X-Z$ plane 


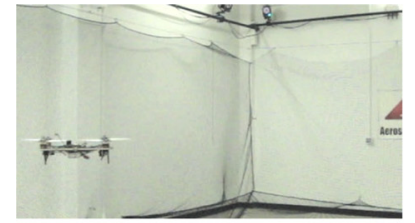

(a)

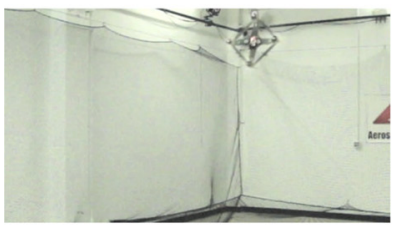

(d)

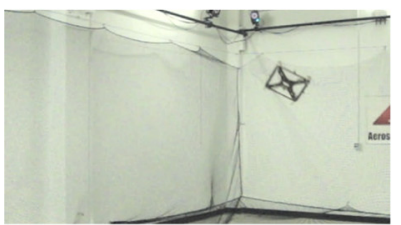

(g)

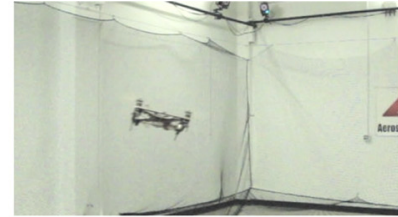

(b)

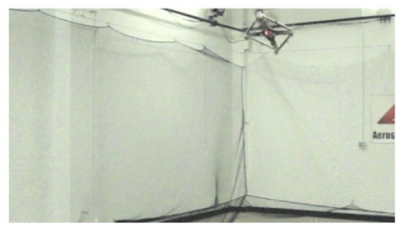

(e)



(h)

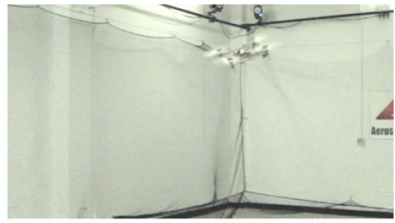

(c)

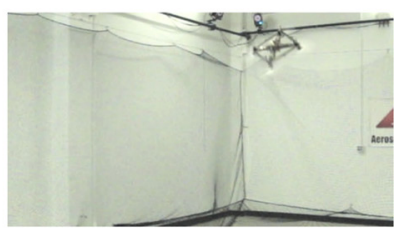

(f)

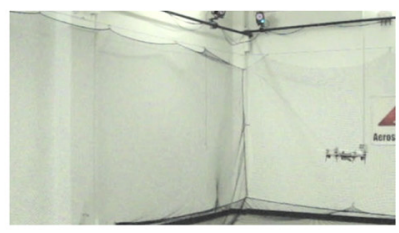

(i)

Fig. 21 Variable-pitch quadrotor performing a 360 deg translating backflip (simulations of this maneuver are in Fig. 11)

Videos of these flight experiments are available under the "Supplemental Data" tab for this paper on the ASME Digital Collection.

\section{Conclusion}

This paper presented the analysis, control, and flight testing of a variable-pitch quadrotor helicopter. Adding variable-pitch propellers to a quadrotor was shown both analytically and experimentally to increase the possible thrust rate of change and provide a quick method for generating reverse thrust. The variable-pitch quadrotor overcomes fundamental limitations of fixed-pitch quadrotors without introducing the mechanical complexity of a pod-and-boom style helicopter. Compared with a fixed-pitch quadrotor, these capabilities greatly increase the possibility for aggressive and aerobatic maneuvers.

A closed-loop flight control algorithm and novel trajectory generation scheme were also developed. The trajectories are generated while accounting for actuator saturation levels and can accept attitude constraints along the path. Some example, maneuvers are performed on a real variable-pitch quadrotor showing increased performance over fixed-pitch quadrotors.

\section{Acknowledgment}

This paper was based upon the work supported by the National Science Foundation Graduate Research Fellowship under Grant No. 0645960. The authors also acknowledge the Boeing Research and Technology for support of the RAVEN indoor flight facility in which the flight experiments were conducted.

\section{References}

[1] Amir, M. Y., and Abbass, V., 2008, "Modeling of Quadrotor Helicopter Dynamics," International Conference on Smart Manufacturing Application (ICSMA), Gyeonggi-do, Apr. 9-11, pp. 100-105.

[2] Erginer, B., and Altug, E., 2007, "Modeling and PD Control of a Quadrotor VTOL Vehicle," IEEE Intelligent Vehicles Symposium, Istanbul, Turkey, June 13-15, pp. 894-899.
[3] Alpen, M., Frick, K., and Horn, J., 2009, "Nonlinear Modeling and Position Control of an Industrial Quadrotor With On-Board Attitude Control," IEEE International Conference on Robotics and Automation, Christchurch, Dec. 10-11, pp. 2329-2334

[4] Kim, J., Kang, M., and Park, S., 2010, “Accurate Modeling and Robust Hovering Control for a Quad-Rotor VTOL Aircraft,” J. Intell. Rob. Syst., 57(1), pp. 9-26.

[5] Huang, H., Hoffmann, G., Waslander, S., and Tomlin, C., 2009, “Aerodynamics and Control of Autonomous Quadrotor Helicopters in Aggressive Maneuvering," IEEE International Conference on Robotics and Automation, Kobe, Japan, May $12-17$, pp. 3277-3282.

[6] Gurdan, D., Stumpf, J., Achtelik, M., Doth, K. M., Hirzinger, G., and Rus, D. 2007, "Energy-Efficient Autonomous Four-Rotor Flying Robot Controlled at $1 \mathrm{kHz}$," IEEE International Conference on Robotics and Automation, Rome, Italy, Apr. 10-14, pp. 361-366.

[7] Michael, N., Mellinger, D., Lindsey, Q., and Kumar, V., 2010, “The GRASP Multiple Micro-UAV Testbed," IEEE Rob. Autom. Mag., 17(3), pp. 56-65.

[8] Gillula, J., Huang, H., Vitus, M., and Tomlin, C., 2010, "Design of Guaranteed Safe Maneuvers Using Reachable Sets: Autonomous Quadrotor Aerobatics in Theory and Practice," IEEE International Conference on Robotics and Automation, Anchorage, AK, May 3-7, pp. 1649-1654

[9] Hoffmann, G., Waslander, S., and Tomlin, C., 2008, "Quadrotor Helicopter Trajectory Tracking Control," AIAA Paper No. 2008-7410

[10] Ritz, R., Hehn, M., Lupashin, S., and D'Andrea, R., 2011, "Quadrocopter Performance Benchmarking Using Optimal Control," IEEE/RSJ International Conference on Intelligent Robots and Systems, San Francisco, CA, Sep. 25-30, pp. 5179-5186.

[11] Mellinger, D., and Kumar, V., 2011, "Minimum Snap Trajectory Generation and Control for Quadrotors," IEEE International Conference on Robotics and Automation, Shanghai, China, May 9-13, pp. 2520-2525.

[12] Shen, S., Mulgaonkar, Y., Michael, N., and Kumar, V., 2013, "Vision-Based State Estimation and Trajectory Control Towards Aggressive Flight With a Quadrotor," Robotics: Science and Systems, pp. 1-8.

[13] Pounds, P., and Mahony, R., 2009 "Design Principles of Large Quadrotors for Practical Applications," IEEE International Conference on Robotics and Automation, Kobe, Japan, May 12-17, pp. 3265-3270.

[14] Kushleyev, A., Mellinger, D., Powers, C., and Kumar, V., 2013, "Towards a Swarm of Agile Micro Quadrotors," Auton. Rob., 35(4), pp. 287-300.

[15] Driessens, S., and Pounds, P. E., 2013, "Towards a More Efficient Quadroto Configuration," IEEE/RSJ International Conference on Intelligent Robots and Systems, Tokyo, Japan, Nov. 3-7, pp. 1386-1392.

[16] Borenstein, J., 1992, "The Hoverbot, an Electrically Powered Flying Robot," http://www.cs.cmu.edu/ motionplanning/papers/sbp_papers/integrated1/borenstein hovercraft.pdf

[17] d'Ambrosio, G., and Navoni, R., "HG3 Willy," You Tube video, 3:21, July 2001, http://youtu.be/M4uXmekZk-4 
[18] Michini, B., Redding, J., Ure, N. K., Cutler, M., and How, J. P., 2011, "Design and Flight Testing of an Autonomous Variable-Pitch Quadrotor," IEEE International Conference on Robotics and Automation, Shanghai, China, May 9-13, pp. 2978-2979.

[19] Chen, H., "Variable-Pitch Quadrotor," You Tube video, 3:17, July 2011, http:// youtu.be/fkSx3fSz0tE

[20] Lupashin, S., and D’Andrea, R., 2012, “Adaptive Fast Open-Loop Maneuver for Quadrocopters,” Auton. Rob., 33(1-2), pp. 89-102.

[21] Mellinger, D., Michael, N., and Kumar, V., 2012, "Trajectory Generation an Control for Precise Aggressive Maneuvers With Quadrotors," Int. J. Rob. Res., 31(5), pp. 664-674.

[22] Muller, M., Lupashin, S., and D'Andrea, R., 2011, "Quadrocopter Ball Juggling," IEEE/RSJ International Conference on Intelligent Robots and Systems, San Francisco, CA, Sep. 25-30, pp. 5113-5120.

[23] Ritz, R., Muller, M., Hehn, M., and D’Andrea, R., 2012, “Cooperative Quadrocopter Ball Throwing and Catching," IEEE/RSJ International Conference on Intelligent Robots and Systems, Vilamoura, Oct. 7-12, pp. 4972-4978.

[24] Hehn, M., and D'Andrea, R., 2011, “A Flying Inverted Pendulum,” IEEE International Conference on Robotics and Automation, Shanghai, China, May 9-13. pp. 763-770.

[25] Abbeel, P., Coates, A., and Ng, A. Y., 2010, "Autonomous Helicopter Aerobatics Through Apprenticeship Learning,” Int. J. Rob. Res., 29(13), pp. 1608-1639.

[26] Cutler, M., Ure, N. K., Michini, B., and How, J. P., 2011, "Comparison of Fixed and Variable Pitch Actuators for Agile Quadrotors," AIAA Paper No. 2011-6406.

[27] Cutler, M., and How, J. P., 2012, "Actuator Constrained Trajectory Generation and Control for Variable-Pitch Quadrotors,” AIAA Paper No. 2012-4777.

[28] Drela, M., 2009, “Qprop Users Guide," http://web.mit.edu/drela/Public/web/qprop/

[29] Hemati, N., and Leu, M., 1992, "A Complete Model Characterization of Brushless DC Motors," IEEE Trans. Ind. Appl., 28(1), pp. 172-180.

[30] Colton, S. W., 2010, "Design and Prototyping Methods for Brushless Motor and Motor Control," Master's thesis, Department of Mechanical Engineering, Massachusetts Institute of Technology, Cambridge, MA.

[31] Bristeau, P., Martin, P., Salaun, E., and Petit, N., 2009, "The Role of Propeller Aerodynamics in the Model of a Quadrotor UAV," European Control Conference, pp. 683-688.

[32] Drela, M., 1989, "Xfoil: An Analysis and Design System for Low Reynold Number Airfoils," Low Reynolds Number Aerodynamics, pp. 1-12, http:// web.mit.edu/drela/Public/web/xfoil/
[33] Kuipers, J. B., 2002, Quaternions and Rotation Sequences: A Primer With Applications to Orbits, Aerospace, and Virtual Reality, Princeton University, Princeton, NJ.

[34] Turpin, M., Michael, N., and Kumar, V., 2012, "Trajectory Design and Control for Aggressive Formation Flight With Quadrotors," Auton. Rob., 33(1-2), pp. $143-156$.

[35] Lupashin, S., Schollig, A., Sherback, M., and D'Andrea, R., 2010, “A Simple Learning Strategy for High-Speed Quadrocopter Multi-Flips,” IEEE International Conference on Robotics and Automation, Anchorage, AK, May 3-7, pp. $1642-1648$.

[36] Hehn, M., and D'Andrea, R., 2011, “Quadrocopter Trajectory Generation and Control," World Congress, Vol. 18, pp. 1485-1491.

[37] Markley, F., 2002, "Fast Quaternion Attitude Estimation From Two Vector Measurements," J. Guid., Control, Dyn., 25(2), pp. 411-414.

[38] Chaturvedi, N., Sanyal, A., and McClamroch, N., 2011, "Rigid-Body Attitude Control," IEEE Control Syst., 31(3), pp. 30-51.

[39] Baruh, H., 1999, Analytical Dynamics, WCB/McGraw-Hill, New York.

[40] Michini, B., 2009, "Modeling and Adaptive Control of Indoor Unmanned Aerial Vehicles," Master's thesis, Department of Aeronautics and Astronautics, Massachusetts Institute of Technology, Cambridge MA.

[41] Wie, B., and Barba, P. M., 1985, "Quaternion Feedback for Spacecraft Large Angle Maneuvers," AIAA J., 8(3), pp. 360-365.

[42] How, J. P., Frazzoli, E., and Chowdhary, G. V., 2015, "Linear Flight Control Techniques for Unmanned Aerial Vehicles," Handbook of Unmanned Aerial Vehicles, Springer, New York, pp. 529-576.

[43] Girish, C. V., Emilio, F., Jonathan, H. P., and Hugh, L., 2015, "Nonlinear Fligh Control Techniques for Unmanned Aerial Vehicles," Handbook of Unmanned Aerial Vehicles, Springer, New York, pp. 577-612.

[44] Van Der Merwe, R., and Wan, E. A., 2004, "Sigma-Point Kalman Filters for Integrated Navigation," 60th Annual Meeting of the Institute of Navigation, pp. 641-654.

[45] Valenti, M., Bethke, B., Fiore, G., How, J. P., and Feron, E., 2006, "Indoor Multi-Vehicle Flight Testbed for Fault Detection, Isolation, and Recovery," AIAA Paper No. 2006-6200.

[46] How, J. P., Bethke, B., Frank, A., Dale, D., and Vian, J., 2008, "Real-Time Indoor Autonomous Vehicle Test Environment," IEEE Control Syst. Mag., 28(2), pp. 51-64 